\title{
TETRAHYDROCANNABINOLIC ACID A (THCA-A) REDUCES ADIPOSITY AND PREVENTS METABOLIC DISEASE CAUSED BY DIET-INDUCED OBESITY
}

Belén Palomares ${ }^{1,2,3}$, Francisco Ruiz-Pino ${ }^{1,2,3}$, Martin Garrido-Rodriguez ${ }^{1,4}$, M. Eugenia Prados $^{5}$, Miguel A. Sánchez-Garrido ${ }^{1,2,3}$, Inmaculada Velasco ${ }^{1,2,3}$, María J. Vazquez ${ }^{1,2,3}$, Xavier $\mathrm{Nadal}^{6}$, Carlos Ferreiro-Vera ${ }^{6}$, Rosario Morrugares ${ }^{1,2,3}$, Giovanni Appendino ${ }^{7}$, Gaetano Morello $^{8}$, Marco A Calzado ${ }^{1,2,3}$, Manuel Tena-Sempere ${ }^{1,2,3, *}$, and Eduardo 10 Muñoz ${ }^{1,2,3, *}$

${ }^{1}$ Instituto Maimónides de Investigación Biomédica de Córdoba (IMIBIC), Córdoba, Spain; ${ }^{2}$ Departamento de Biología Celular, Fisiología e Inmunología, Universidad de Córdoba, Córdoba, Spain; ${ }^{3}$ Hospital Universitario Reina Sofía, Córdoba, Spain; ${ }^{4}$ Innovative Health 15 Group, Madrid; ${ }^{5}$ Emerald Health Biotechnology España, Córdoba, Spain: ${ }^{6}$ Phytoplant Research, Córdoba, Spain; ${ }^{7}$ Dipartimento di Scienze del Farmaco, Università del Piemonte Orientale, Novara, Italy; ${ }^{8}$ Emerald Health Naturals, Vancouver, Canada.

*Senior authors; contributed equally.

Corresponding author: Eduardo Muñoz, MD, PhD. Maimonides Biomedical Research Institute of Córdoba, University of Córdoba, Avda Menéndez Pidal s/n. 14004. Córdoba, Spain. Phone: +34 957213766. Email: fi1muble@uco.es 


\section{ABSTRACT}

Cannabis has remarkable therapeutic potential, but its clinical use is limited by the psychotropic activity of $\Delta^{9}$-tetrahydrocannabinol $\left(\Delta^{9}\right.$-THC). Surprisingly, the biological profile of the non-narcotic native precursor of $\Delta^{9}$-THC $\left(\Delta^{9}\right.$-THC acid A, $\Delta^{9}$-THCA-A $)$ is still largely unexplored. We present evidence that $\Delta^{9}$-THCA-A is a partial and selective PPAR $\gamma$ modulator, endowed with lower adipogenic activity than the full PPAR $\gamma$ agonist rosiglitazone (RGZ) and with an enhanced osteoblastogenic activity in human mesenchymal stem cells. Docking and in vitro functional assays indicated that $\Delta^{9}$-THCA-A binds to and activates PPAR $\gamma$ by acting at both the canonical and the alternative sites of the ligand-binding domain. Transcriptomic signatures at inguinal white adipose tissue (iWAT) from mice treated with $\Delta^{9}$ THCA-A confirmed its mode of action on PPAR $\gamma$. Administration of $\Delta^{9}$-THCA-A in a mouse model of high fat diet (HFD)-induced obesity significantly reduced fat mass and body weight gain, markedly ameliorating glucose intolerance and insulin resistance, and largely preventing liver steatosis, adipogenesis and macrophage infiltration in fat tissues. Additionally, immunohistochemistry, transcriptomic, and plasma biomarker analyses showed that treatment with $\Delta^{9}$-THCA-A caused browning of iWAT and displayed potent anti-inflammatory actions in HFD mice. Altogether, our data validate the potential of $\Delta^{9}$-THCA-A as a low adipogenic PPAR $\gamma$ agonist, capable of substantially improving the symptoms of obesity-associated metabolic syndrome and inflammation. These findings suggest that $\Delta^{9}$-THCA-A, and perhaps non-decarboxylated Cannabis sativa extracts, are worth considering for addition to our inventory of cannabis medicines.

Key words: Medicinal cannabis, $\Delta^{9}$-THCA-A, PPAR $\gamma$, obesity, metabolic syndrome, inflammation 


\section{SIGNIFICANCE STATEMENT}

The medicinal use of Cannabis is gaining momentum, despite the adverse psychotropic effects of $\Delta^{9}$-THC, the decarboxylation product of its naturally occurring and nonpsychotropic precursor $\Delta^{9}$-THCA-A. We present evidence that $\Delta^{9}$-THCA-A is a partial ligand agonist of PPAR $\gamma$ with lower adipogenic activity compared to the full PPAR $\gamma$ agonist rosiglitazone (RGZ). Moreover, chronic administration of $\Delta^{9}$-THCA-A in a mouse model of high fat diet (HFD)-induced obesity significantly reduced body weight gain and fat mass, improved glucose intolerance and insulin resistance, and prevented liver steatosis and macrophage infiltration in fat tissues, additionally inducing white adipose tissue browning.

60 Collectively, these observations qualify $\Delta^{9}$-THCA-A, a compound devoid of psychotropic effects, as an efficacious pharmacological agent to manage metabolic syndrome and obesityassociated inflammation. 


\section{Highlights}

- $\Delta^{9}$-THCA-A is a partial PPAR $\gamma$ ligand agonist with low adipogenic activity

- $\Delta^{9}$-THCA-A enhances osteoblastogenesis in bone marrow derived mesenchymal stem cells.

$70-\Delta^{9}$-THCA-A reduces body weight gain, fat mass, and liver steatosis in HFD-fed mice

- $\Delta^{9}$-THCA-A improves glucose tolerance, insulin sensitivity, and insulin profiles in vivo

- $\Delta^{9}$-THCA-A induces browning of iWAT and has a potent anti-inflammatory activity 


\section{INTRODUCTION}

Preparations of Cannabis sativa have been used since the earliest written records of medical history, complementing the nutritional and technological uses of the plant and contributing to make it one of the first species cultivated by humans. Modern studies as well as anecdotal reports suggest the potential efficacy of cannabis extracts and cannabinoids in different medical conditions. Almost 150 cannabinoids have been isolated from different strains of Cannabis (1) with $\Delta^{9}$-tetrahydrocannabinol $\left(\Delta^{9}\right.$-THC) and cannabidiol (CBD) being the best investigated class members from a medical standpoint. These neutral cannabinoids are produced and stored in the plant as acidic precursors (cannabinoid acids) (2). Decarboxylation requires heating but can take place also at room temperature upon prolonged storage of Cannabis (3). Remarkably, the acidic precursor of $\Delta^{9}$-THC ( $\Delta^{9}$-THCA-A) is not psychotropic, and its binding to cannabinoid receptors is highly controversial and probably associated to contamination by its decarboxylation product (4). On the other hand, we recently showed that THCA-A, but not its decarboxylation product $\Delta^{9}-\mathrm{THC}$, is a potent activator of the peroxisome proliferator-activated receptor- $\gamma(\operatorname{PPAR} \gamma)$ endowed with remarkable neuroprotective activity (5). In agreement with these findings, the PPAR $\gamma$ activating properties of a Cannabis extract with a high titer of $\Delta^{9}$-THC was greatly reduced by decarboxylation (5). Given the key metabolic roles of PPAR $\gamma$, these findings provided a rationale to investigate the possibility that $\Delta^{9}$-THCA-A based therapies could be beneficial for the management of metabolic disorders.

Obesity has reached pandemic proportions worldwide, with more than 650 million obese and nearly 2 billion people being overweighed (6). Obesity is a major risk factor for the development of multiple diseases, including prominently cardiovascular and metabolic disorders, such as type-2 diabetes and metabolic syndrome (MetS) $(7,8)$, but also respiratory, osteoarticular, cognitive, reproductive and oncologic pathologies (9-12). The basis for the 100 multi-organ alterations seen in obesity remains unfolded (13), but the state of chronic, lowgrade inflammation commonly associated to this condition is considered a major contributing factor (11). This is reflected by the increased concentrations of white adipose tissue-derived pro-inflammatory cytokines (TNF- $\alpha$, IL-6, leptin) and the antiparallel decrease of antiinflammatory signals, as adiponectin, commonly observed in obesity $(12,14,15)$. Additional hormonal perturbations, e.g., of gastro-intestinal factors, such as ghrelin and GLP-1 (16), as well as the ectopic deposition of fat, mostly in the liver (i.e., steatosis), which defines a state of lipotoxicity (17), contribute also to the worsening of the metabolic profile of obese 
patients. In view of the conspicuous lack of effective therapies for the integral management of obesity, considerable attention is currently given to the identification of novel pharmacological agents globally targeting its deregulated substrates and other related pathways, as thermogenesis, to regain energy homeostasis (18-20).

In fact, while initial strategies to tackle obesity were mostly focused in the control of food intake, growing interest has been paid recently to elucidate the physiological roles and eventual therapeutic use of adaptive thermogenesis in the control of body weight and energy 115 homeostasis $(21,22)$. Studies in this area have been directed not only to the brown adipose tissue (BAT), where canonical adaptive thermogenesis occurs in mammals, including humans (23), but also to the capacity of the white adipose tissue (WAT) to undergo, under certain conditions, browning, a process whereby adipocyte precursor cells acquire brown-like features, becoming beige or brite adipocytes (24). Brown and beige adipocytes are defined by a large set of mitochondria and high expression of uncoupling protein-1 (UCP-1), as major molecular switch to enhance heat dissipation at the expense of lower ATP synthesis at the respiratory chain (25). Thermogenic activity is finely regulated by the direct actions of a large set of circulating hormones, from adipokines to sex steroids and thyroid hormones $(23,26)$, as well as the adrenergic input from the sympathetic nervous system (27), therefore being amenable for pharmacological manipulation.

PPAR $\gamma$ is a nuclear receptor that plays key role in regulating a number of biological functions including lipid and glucose metabolism (28). PPAR $\gamma$ is also involved in the natural and adaptive immune response as well as in additional biological activities like the browning of white fat (29). PPAR $\gamma$ ligands include a wide array of natural and synthetic molecules, with

130 glitazones, a class of compounds extensively for the management of type-2 diabetes, being the archetypal activators. Glitazones, like rosiglitazone (RGZ), are considered full PPAR $\gamma$ ligand agonists, and this profile is inextricably associated to both antidiabetic activity and undesirable side effects like weight gain, edema, and osteoporosis (30). Furthermore, liver injury, cancer, as well as an increased risk of heart failure have also been associated with the 135 long-term use of glitazones (30). However, while the therapeutic potential of PPAR $\gamma$ ligand agonist remains high, interest has substantially shifted towards partial ligands, preeminent examples of which are natural products such as armofrutins and acidic cannabinoids $(5,31)$.

Depending on the mode of interaction and binding to the ligand-binding domain (LBD) of PPAR $\gamma(32,33)$, modulators can induce graded responses, such as full- and partial agonism and antagonism. Thus, the recent identification of a functional alternative binding site for 
PPAR $\gamma$ ligands that does not fully overlap with the canonical glitazone binding site (34) has opened novel research avenues for the identification and/or development of second-generation PPAR $\gamma$ agonists.

\section{RESULTS}

\section{$\Delta{ }^{9}$-THCA-A is a selective and non-adipogenic PPAR $\gamma$ ligand agonist that induces iWAT browning through a PPAR $\gamma$-dependent pathway}

We have previously shown that $\Delta^{9}$-THCA-A is a PPAR $\gamma$ agonist at nanomolar concentrations (5). Herein, we have studied the selectivity of $\Delta^{9}$-THCA-A on different PPARs, showing that, when compared to the full ligand agonist rosiglitazone (RGZ), $\Delta^{9}$ THCA-A is only a partial ligand activator for PPAR $\gamma$, devoid of PPAR $\alpha$ and PPARd transcriptional activities (Fig. 1A). Dephosphorylation of PPAR $\gamma$ at Ser273 is essential for acquiring insulin-sensitizing activity (35), and we found that, in 3T3-L1 adipocytes, $\Delta^{9}$-THCA-A, as well as RGZ, could inhibit the TNF $\alpha$-induced Ser273 phosphorylation of PPAR $\gamma($ Fig. 1B).

PPAR $\gamma$ has a large and dynamic LBD, whose diversity of ligand accommodation in its two sub-pockets is associated to distinct biological activities (32). Based on the structure of different PPAR $\gamma$-ligand complex, it has been proposed that full agonists bind to both subpockets, establishing hydrogen bonds with residues Tyr473 (H12) on AF-2 (also called orthosteric or canonical binding site) and Ser342 (S1/S2) on $\beta$-sheet sub-pocket (also called alternative binding site), whereas partial agonists can preferentially bind to the alternative site (34). Docking analysis suggested that $\Delta^{9}$-THCA-A binds both the canonical (Ser289) and the alternative binding sites (L340 and Ser342) in LBD (Fig. S1). The functionality of both LBD

165 sites in the response to $\Delta^{9}$-THCA-A was therefore investigated. To this purpose, T0070907, a $\operatorname{PPAR} \gamma$ antagonist that binds irreversibly to the canonical binding site, was used. First, luciferase reporter assays were used to study the participation of the canonical and alternative binding sites in the response to $\Delta^{9}$-THCA-A in comparison with RGZ. As expected, preincubation with T0070907 effectively blocked RGZ-induced PPAR $\gamma$ transactivation (Fig.

170 1C). Conversely, T0070907 could not block $\Delta^{9}$-THCA-A-induced PPAR $\gamma$ transcriptional activity (Fig. 1D). In addition, T0070907 almost fully suppressed the expression of PPAR $\gamma$ dependent genes induced by RGZ in human mesenchymal stem cells (MSCs) differentiated to adipocytes (Fig. 1E). Conversely, T0070907 did not fully prevent the effect of $\Delta^{9}$-THCA-A 
on these genes (Fig. 1F). By using time-resolved fluorescence resonance energy transfer (TR-

175 FRET) co-regulator interaction assays, we found that RGZ increases the binding of peptides derived from TRAP220 and PGC-1 $\alpha$, but decreases the binding of peptides derived from NCoR and SMRT. In contrast, $\Delta^{9}$-THCA-A has not significant effect on the recruitment TRAP220 to PPAR $\gamma$ and showed only a moderate effect on the recruitment of PGC$1 \alpha$. Indeed, $\Delta^{9}$-THCA-A had a weak effect to displace corepressors from the binding to PPAR $\gamma$ (Fig. S2).

To further investigate the role of the PPAR $\gamma$ canonical binding site in vivo, mice were treated with $\Delta^{9}$-THCA-A in the presence or not of T0070907, performing transcriptomic analysis in inguinal white adipose tissue (iWAT), a major target organ for PPAR $\gamma$ agonists. The comparative differential expression analysis of both conditions versus control mice resulted in a total of 3719 genes with an adjusted $\mathrm{P} \leq 0.01$ and an absolute fold change $\geq 2$ (Fig. 2A). From them, 72 genes belonging to the PPAR $\gamma$ signaling and thermogenesis pathways were upregulated in response to $\Delta^{9}$-THCA-A (Fig. 2A-B). Within this group of genes, the co-treatment with T0070907 prevented the upregulation of 46 genes induced by $\Delta^{9}$ THCA-A and 26 genes were unaffected (Fig. 2C). In contrast, genes included in the NF-кB signaling pathway were not affected by $\Delta^{9}$-THCA-A treatment, but significantly increased their expression in animals co-treated with T0070907 (Fig. 2A-B). Uncoupling protein 1 (UCP-1) gene, a key marker of the iWAT browning process, was found among the top 25 genes upregulated by $\Delta^{9}$-THCA-A in a T0070907-sensitive manner (Fig. 2D). Moreover, western blotting and immunohistochemistry showed that $\Delta^{9}$-THCA-A induced the expression of UCP-1 protein in iWAT (Fig. 2E). In line with such putative thermogenic activation, $\Delta^{9}$ THCA-A treatment caused a suppression of body weight that was independent of food intake changes but blocked by co-administration of T0070907 (Fig. S3).

In our analysis, we also found 317 genes that were modified by $\Delta^{9}$-THCA-A in a way insensitive to T0070907 (Fig. S4A). To functionally evaluate this cluster of genes, we performed an over-representation analysis using the KEGG pathways and Gene Ontology annotations after sorting them out into up- and down-regulated ones (Fig. S4B-C). Interestingly, a set of upregulated genes associated to fatty acid metabolism was found. Conversely, genes belonging to cGMP-PKG, to calcium signaling pathways, and to muscle tissue development were downregulated by $\Delta^{9}$-THCA-A administration. Taken together, our results showed that the bioactivity of $\Delta^{9}$-THCA-A was mediated by the PPAR $\gamma$ canonical 
pathway as well as by pathways mediated by the alternative binding site of this nuclear receptor.

PPAR $\gamma$ partial ligand agonists are thought to be less adipogenic than full ligand agonists and are also expected to have fewer negative effects on bone metabolism. Therefore, we studied the ability of $\Delta^{9}$-THCA-A to influence MSCs differentiation into adipocytes and osteoblasts. MSCs were cultured in adipogenic medium (AM) for either 14 days or 21 days to evaluate the mRNA expression of adipogenic markers and to detect lipid droplets. Fig. 3A-B show that MSC treated with $\Delta^{9}$-THCA-A contained fewer and smaller lipid droplets compared to RGZ treatment. Moreover, $\Delta^{9}$-THCA-A induced lower expression of the adipogenic differentiation markers PPAR $\gamma, \mathrm{aP} 2 \mathrm{a}, \mathrm{ADIPOQ}, \mathrm{LPL}$ and CEBPA, as compared to cells treated with RGZ (Fig. 3C). Remarkably, we found that, in MSC differentiated in an osteoblastogenic medium, $\Delta^{9}$-THCA-A enhanced osteoblast mineralization as well as the expression of the osteogenic differentiation markers Runx2, SP7, IBS and ALP, (Fig. 3D-F). These data indicate that $\Delta^{9}$-THCA-A qualifies as a partial PPAR $\gamma$ ligand significantly less adipogenic than RZG and with an enhanced osteoblasts differentiation capacity.

\section{$\Delta^{9}$-THCA-A treatment ameliorates HFD-induced metabolic perturbations and iWAT inflammation}

In addition, we analyzed the effects of chronic treatment for 3-wks with an effective dose of $\Delta^{9}$-THCA-A $(20 \mathrm{mg} / \mathrm{kg} \mathrm{BW} /$ day $)$ on various metabolic and hormonal parameters in a mouse model of HFD-induced obesity. Feeding a HFD for 12-wks resulted in a significant increase in body weight over CD controls (BW; Fig. 4A), together with enhanced fat mass $(35.89 \pm 2.63 \mathrm{~g}$ vs. $16.19 \pm 1.45 \mathrm{~g}$ in $\mathrm{CD} ; \mathrm{P}>0.001)$ and adiposity index, calculated as ratio between fat mass and fat + lean mass $(36.43 \pm 2.62$ vs. $16.56 \pm 1.49 \%$ in $\mathrm{CD} ; \mathrm{P}=0.001)$.

230 Repeated administration of $\Delta^{9}$-THCA-A caused a marked suppression of BW gain in HFD mice (Fig. 4B), as observed, albeit with lesser amplitude, in CD mice. The increased adiposity caused by HFD was similarly restored by chronic $\Delta^{9}$-THCA-A treatment, which caused also a significant suppression of the adiposity index in control lean mice (Fig. 4C).

Administration of $\Delta^{9}$-THCA-A for 3-wks improved also glucose homeostasis in HFD235 induced obese mice. Thus, while HFD exposure for 15-wks evoked an elevation of basal glucose levels, worsened glucose tolerance after glucose bolus injection (Fig. 4D) and reduced insulin sensitivity (Fig. 4E), treatment of HFD mice with $\Delta^{9}$-THCA-A for 3-wks resulted in lowering of basal glycemia, and markedly improved glucose profiles, both in 
glucose and insulin tolerance tests, which displayed better profiles than those of CD mice without pharmacological intervention. Moreover, positive effects of $\Delta^{9}$-THCA-A in terms of glucose tolerance and insulin sensitivity were also detected in lean control mice (Fig. 4D-E). This was associated to a significant lowering of basal insulin levels after $\Delta^{9}$-THCA-A administration to CD and HFD mice (Fig. 4F), possibly reflecting a state of enhanced insulin sensitivity. In the same line, $\Delta^{9}$-THCA-A treatment of obese mice largely prevented liver fat 245 infiltration caused by HFD and markedly reduced the steatosis score (Fig. 4G-H). In good agreement with these observations, $\Delta^{9}$-THCA-A significantly decreased serum triglyceride levels both in obese and lean mice (Fig. 4I).

The iWAT transcriptomic profile in control and HFD mice, untreated or treated with $\Delta^{9}$ THCA-A, was next investigated. A differential expression analysis revealed a total of 1387 genes overcoming the cutoff of an adjusted $\mathrm{P} \leq 0.01$ and an absolute fold change $\geq 2$ in any of the two comparisons (Fig. 5A). Among them, KEGG pathway analyses revealed that genes involved in NF- $\kappa$ B signaling and cytokine-cytokine receptor were upregulated in HFD mice, which matches the inflammatory phenotype in iWAT that accompanies the metabolic syndrome (36). Additionally, genes belonging to the insulin receptor signaling pathway showed a lowered expression in HFD mice, in agreement with the hallmark state of insulin resistance of obesity. Interestingly, $\Delta^{9}$-THCA-A treatment in HFD mice reduced the expression of genes belonging to the inflammatory pathways, partially recovering the expression of those linked to the insulin signaling process (Fig. 5A-B). In total, DEGs analysis revealed that 1014 genes including those related to NF- $\kappa \mathrm{B}$ signaling and cytokinecytokine receptor (70 genes) were modified in HFD mice and normalized in $\Delta^{9}$-THCA-Atreated HFD mice (Fig. 5C). Finally, to confirm the anti-inflammatory profile of $\Delta^{9}$-THCA-A, we analyzed by qPCR assays the top 25 upregulated inflammatory genes in the iWAT of HFD mice (Fig. 5D), confirming the increased expression of key components of this gene set, including TNF $\alpha$, ICAM-1, CD4, CXCL-16, CCK22, CXCR5 and CXCR2 (Fig. 5E).

In keeping with their obese phenotype, HFD mice also displayed features of adipose tissue enlargement and inflammation. Thus, a significant increase in adipocyte volume was observed, accompanied by signs of macrophage infiltration of white adipose tissue (WAT), assessed by F4/80 staining of clusters of macrophages surrounding dead adipocytes, in the socalled crown-like structures (CLS) (Fig. 6A-B). Notably, $\Delta^{9}$-THCA-A administration for 3wks fully prevented adipocyte enlargement and the appearance of CLS in the inguinal WAT 
of HFD-induced obese mice. Moreover, a substantial upsurge in the levels of the thermogenic protein, UCP-1, in the iWAT of obese mice was also observed (Fig. 6A-C).

Finally, changes in the circulating levels of key metabolic hormones were assessed in HFD-induced obese mice, treated or not with $\Delta^{9}$-THCA-A. In line with their obese phenotype, HFD mice showed increased leptin and glucagon levels, and decreased GLP-1 concentrations vs. lean CD animals. Treatment with $\Delta^{9}$-THCA-A for 3-wks fully normalized leptin, GLP-1 and glucagon levels, and evoked a significant increase in adiponectin concentrations (Fig. 6DG). In the same vein, proteome profiler arrays targeting a comprehensive set of circulating adipokines and cytokines confirmed the increase of the levels of leptin, together with other adipose born-factors, such as Oncostatin M and Serpin E1, in HFD mice, which were decreased by $\Delta^{9}$-THCA-A, while adiponectin concentrations were increased. Likewise, a set of cytokines including Endostatin, IGFBp-5, PCSK9, Adipsin and IGFBP-3 were suppressed by HFD but increased by $\Delta^{9}$-THCA-A in HFD mice, while an opposite pattern was observed for CCL11 and CCL6 (Fig. 6I).

\section{DISCUSSION}

Because of its escalating prevalence and the enormous health and economic burden associated to its co-morbidities, such as type 2 diabetes, obesity has become a major societal problem, now recognized as the major preventable risk factor for all-cause mortality $(6,37)$, with a nearly 50\% more life-years lost compared to other major avertable factors, like smoking. Glitazones, such as RGZ, are synthetic full PPAR $\gamma$ agonists that have been marketed since the early 2000s for the treatment of type 2 diabetes. However, the use of glitazones in diabetic patients has dropped significantly over the past years due to a series of adverse side effects that include bone loss and osteoporosis, as well as fluid retention. Bone loss could be bound to the effect of glitazones on the lineage commitment of mesenchymal stem cells towards osteoblasts and adipocytes, and it has been shown that RGZ suppresses osteoblastogenesis and induces adipocyte differentiation (38). The development of more balanced partial PPAR $\gamma$ agonists, devoid of the side effects showed by the currently marketed PPAR $\gamma$ full agonists, is considered a major challenge for drug discovery (39). We provide evidence that $\Delta^{9}$-THCA-A is a partial PPAR $\gamma$ agonist, lacking the adverse psychotropic effects of $\Delta^{9}$-THC, with a lower adipogenic capacity than glitazones, and endowed also with osteoblastogenesisenhancing properties. In in vivo animal experiments, $\Delta^{9}$-THCA-A could successfully prevent 
adiposity and reversed the metabolic and inflammatory complications associated to dietinduced obesity.

Docking and functional analysis revealed that $\Delta^{9}$-THCA-A may exert its PPAR $\gamma$ activity by acting at both the canonical and the alternative binding sites of the PPAR $\gamma$ LBD. $\Delta^{9}$ THCA-A binds to the alternative site by interacting with Ser342 at the $\beta$-sheet ( $\Omega$-loop) through its carboxylate group. This may explain the observation that $\Delta^{9}$-THCA-A is at least 20-fold more potent than $\Delta^{9}$-THC to activate PPAR $\gamma$ (5). In addition, dietary long- and medium-chain fatty acids, as well as small molecules, also bind to the same amino acid in the alternative site, highlighting the relevance of this site for a wide class of synthetic and natural compounds including acidic cannabinoids $(40,41)$. Our data also suggest that $\Delta^{9}$-THCA-A may stabilize the $\Omega$-loop region and inhibit phosphorylation of PPAR $\gamma$ Ser273, thus inducing anti-diabetic activity. However, $\Delta^{9}$-THCA-A does not affect PPAR $\gamma$ co-regulators interaction, and we cannot discard that $\Delta^{9}$-THCA-A could also act as a neutral ligand at the orthosteric site, since most of its activities are inhibited by T0070907. Thus, $\Delta^{9}$-THCA-A could compete with RGZ in PPAR $\gamma$-dependent transcriptional assays (5), and TR-FRET assays also showed that $\Delta^{9}$-THCA-A partially prevented the recruitment of TRAP220 and SMRT induced by RGZ. In this model, $\Delta^{9}$-THCA-A could push endogenous fatty acids out of the orthosteric pocket toward the alternative binding site (40). Nevertheless, it has been shown that others partial agonists such as INT131 and BVT-13 do not induce significant binding or displacement of PPAR $\gamma$ co-regulators $(32,41,42)$. Interestingly, SR2595, an inverse PPAR $\gamma$ agonist that blocks phosphorylation of Ser273, could promote osteoblastogenesis in cultured hMSCs (43). In summary, our in vitro and in vivo data strongly suggest that the binding of $\Delta^{9}$ THCA-A to the PPAR $\gamma$ orthosteric site accounts for most of the $\Delta^{9}$-THCA-A effects mediated by this nuclear receptor, but signaling through the alternative site could also be of biological relevance. Thus, in lean mice, 317 genes modified by $\Delta^{9}$-THCA-A but unaffected by T0070907 were found. Among them, upregulation of genes related with the fatty acid metabolism and downregulation of genes belonging to cGMP-PKG, calcium signaling pathways and muscle tissue development, were identified. Whether this pathway is linked or not to the PPAR $\gamma$ alternative site or to other pathways, such as $\mathrm{CB}_{1}$ signaling, remains to be investigated.

There is growing evidence of a link between obesity and inflammation, and $\Delta^{9}$-THCA-A 335 showed a very potent anti-inflammatory profile in HFD mice. Thus, activation of innate and adaptive immune response has been observed in the fat tissue in obese individuals (44-46), 
and we identified 70 upregulated genes linked to NF-кB and cytokine-cytokine-receptor signaling, whose expression was largely prevented by treatment with $\Delta^{9}$-THCA-A. Within them, T- and B cell markers, as well as macrophage-derived cytokines and chemokines, were identified, showing that both the innate and the adaptive immune responses account for the inflammatory status in the fat tissue of HFD mice. There is strong evidence that PPAR $\gamma$

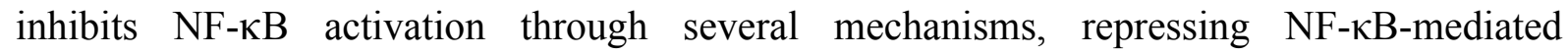
transcription of proinflammatory cytokines in immune and non-immune cells (47). Thus, $\Delta^{9}$ THCA-A could dampen inflammation mainly through the PPAR $\gamma$ pathway, and this phytocannabinoid deserves consideration for the management of other chronic or acute inflammatory diseases.

The efficacy of $\Delta^{9}$-THCA-A to alleviate a wide spectrum of metabolic and hormonal derangements linked to diet-induced obesity was evaluated in a validated preclinical model of metabolic syndrome, namely, 12-wk exposure to HFD (48). This model fully recapitulates the cardinal manifestations of obesity and its major complications, including an increase of body weight, fat mass, adipocyte area and adiposity index, accompanied by perturbed glucose homeostasis and insulin resistance, as well as enhanced liver lipid deposition and steatosis. Notably, a regimen of 3 -wk treatment with an effective daily dose of $\Delta^{9}$-THCA-A was sufficient to reverse such adverse metabolic profile, causing a marked suppression of body weight gain and adiposity, significantly lowering basal glucose and insulin levels, and fully preventing HFD-induced glucose intolerance and insulin resistance. Moreover, circulating triglyceride levels were reduced and steatosis scores were substantially improved by 3 -wk $\Delta^{9}$ THCA-A treatment in HFD mice, substantially reversing the metabolic phenotype caused by the high fat content diet. Likewise, the major hormonal and cytokine alterations caused by 360 HFD were reversed by $\Delta^{9}$-THCA-A, with a significant decrease in circulating leptin and glucagon, and a significant increase in serum GLP-1 and adiponectin levels. Such a switch towards an anti-inflammatory state was also detected by our adipo-/cytokine arrays, which confirmed the reversal of most of the pro-inflammatory humoral alterations caused by HFD. Interestingly, the beneficial effects of $\Delta^{9}$-THCA-A were not only observed in HFD conditions, 365 but also, albeit to a lesser extent, in lean animals fed a control diet, in which 3-wk treatment with $\Delta^{9}$-THCA-A was capable to reduce body weight and adiposity, as well as basal glucose, insulin and triglyceride levels, together with a significant enhancement of glucose tolerance and insulin sensitivity. All these features define an optimal pharmacological profile of $\Delta^{9}$ THCA-A for globally handling the metabolic syndrome linked to obesity. 
The beneficial effects of $\Delta^{9}$-THCA-A on body weight and metabolic status are somewhat at odds with the reported orexigenic and lipogenic activity of cannabinoids $(49,50)$. In our studies, $\Delta^{9}$-THCA-A failed to cause any detectable change in food intake, supporting a distinct pharmacological profile compared to $\Delta^{9}$-THC, whose feeding-promoting effects underlie its use in the management of cachexia (51). The lack of orexigenic effect together with the substantial body weight loss induced by $\Delta^{9}$-THCA-A suggests a potential use of this compound to induce adaptive thermogenesis. Indeed, chronic treatment with $\Delta^{9}$-THCA-A caused the upregulation of a large set of genes belonging of the thermogenesis pathway, with a prominent increase in the UCP-1 content in inguinal WAT that was largely prevented by cotreatment with the PPAR $\gamma$ inhibitor, T0070907. UCP-1 induction was also detected in the WAT of HFD mice. Altogether, these findings suggest that, acting at least partially via PPAR $\gamma, \Delta^{9}$-THCA-A causes browning of the white adipose tissue, and this activity could mechanistically underlie the beneficial effects of this carboxylated cannabinoid on energy and metabolic homeostasis. These features add to our current efforts to identify pharmacological agents capable to activate thermogenesis without undesired side effects.

The global challenge posed by obesity and the inherent difficulties to handle its multifactorial pathophysiology have fueled the search for novel therapeutic agents endowed with multi-target activity and capable to improve the different metabolic alterations associated to overweight. Recent efforts in this area include the development of novel hormonal multiagonists based on peptide chimeras, or on conjugates fusing peptides and small molecules, capable to jointly target the various signaling pathways involved in the pathogenesis of the major complications of obesity (20). While this approach holds the promise to improve obesity treatments, it is not devoid of potential side effects and the pharmacodynamic limitations linked to the integration of different hormonally-active moieties with, in some cases, opposite biological activities. In the context of a poly-pharmacological approach to obesity and metabolic syndrome, the phytocannabinoid, $\Delta^{9}$-THCA-A, deserves further studies, since it is endowed with the beneficial effects of PPAR $\gamma$ agonists but devoid of their adipogenic activity and of the adverse psychotropic and orexigenic effects of narcotic cannabinoids. The effects of the administration of $\Delta^{9}$-THCA-A in a preclinical model of dietinduced obesity equal, if not outperform, the results reported for promising poly-agonist therapies recently advocated for obesity treatment (18-20), suggesting that this nonpsychotropic phytocannabinoid, as well as non-decarboxylated Cannabis sativa extracts, are worth of consideration for the management of obesity and metabolic disease. 


\section{Methods}

405

\section{$\triangle^{9}-T H C A-A$ isolation}

$\Delta^{9}$-THCA-A was purified at $>95 \%$ from the proprietary Cannabis variety Moniek (CPVO/20160114) using a Counter Current Chromatography (CCC) by Phytoplant Research S.L. (Córdoba, ES). An Agilent liquid chromatography set-up (Model 1260, Pittsburgh, PA,

United States) consisting of a binary pump, a vacuum degasser, a column oven, an autosampler and a diode array detector (DAD) equipped with a $150 \mathrm{~mm}$ length, $2.1 \mathrm{~mm}$ internal diameter, $2.7 \mathrm{~mm}$ pore size Poroshell 120 EC-C18 column was used for the quality control of the purified cannabinoids. The analysis was performed using water and acetonitrile both containing ammonium formate $50 \mathrm{mM}$ as mobile phases. Flow-rate was $0.2 \mathrm{~mL} / \mathrm{min}$ and the injection volume was $3 \mu \mathrm{L}$. Chromatographic peaks were recorded at $210 \mathrm{~nm}$. All determinations were carried out at $35^{\circ} \mathrm{C}$. All samples were analyzed in duplicate. The result of $\Delta^{9}$-THCA-A purity $95.42 \%$ and THC impurity $1.32 \%$ was calculated as weight (\%) versus a commercial standard from THC Pharm GmbH (Frankfurt, Germany) and Cerilliant (Round Rock, Texas, USA).

\section{Cell lines and luciferase assays}

HEK-293T and 3T3-L1 cells cells were cultured in Dulbecco's Modified Eagle's Medium (DMEM) supplemented with $10 \%$ FBS, $2 \mathrm{mM}$ L-glutamine and $1 \%$ (v/v) penicillin/streptomycin (Sigma-Aldrich, USA) and maintained at $37^{\circ} \mathrm{C}$ and $5 \% \mathrm{CO}_{2}$ in a 425 humidified atmosphere. HEK-293T $\left(1 \times 10^{5}\right)$ cells were seeded in 24-well plates and transiently co-transfected with the indicated constructs (GAL4-PPAR $\gamma$, GAL4-PPAR $\delta$, GAL4-PPAR $\alpha$ and GAL4-luc) using Roti(C-Fect (Carl Roth, Karlsruhe, Germany). After treatments, the luciferase activities were measured using Dual-Luciferase Assay (Promega, Madison, WI, USA).

\section{In vitro adipocyte and osteoblast differentiation}

Human mesenchymal stem cells (MSCs) derived from bone marrow were seeded in $\alpha$-MEM containing 10\% FBS, $2 \mathrm{mM}$ Glutamine, $1 \mathrm{ng} / \mathrm{ml} \mathrm{bFGF}$, and antibiotics, and adipocyte (AD) and osteoblast (OM) differentiation was performed as described (48). Treatment with RGZ (1

$435 \mu \mathrm{M})$ and THCA-A $(1,5$ and $10 \mu \mathrm{M})$ in the presence and the absence of T0070907 $(5 \mu \mathrm{M})$ started at the same time as the differentiation process. After 7 or 14 days of differentiation, the mRNA was analyzed by qPCR and, after 21 days, adipogenesis and osteoblastogenesis were analyzed by Oil red $\mathrm{O}$ and Alizarin red staining respetively. The lipid accumulation and 
mineralization was quantified by removing the staining solution and absorbance was read at 540 and $405 \mathrm{~nm}$, respectively.

\section{Animal studies}

Six-week old male C57BL6 mice, obtained from Charles Rivers Laboratories (l'Arbresle, France), were pair-housed under constant conditions of light (12 hours of light/dark cycles) and temperature $\left(22 \pm 2{ }^{\circ} \mathrm{C}\right)$, with free access to food (see below) and water. All procedures concerning animal use were reviewed and approved by the Ethics Committee of the University of Cordoba and carried out in accordance with European Union Directive 2010/63/EU for the use and care of experimental animals.

At 8 weeks of age, mice were randomly assigned in two groups $(\mathrm{N}=20)$ and fed either a high-fat diet (HFD), D12451 (Research Diets, New Brunswick, NJ, USA; 45\%, 20\%, and $35 \%$ calories from fat, protein and carbohydrate, respectively) or a standard diet (CD) (A04 SAFE Diets, Augy, France; 8,4\%, $19,3 \%$ and $72,4 \%$ calories from fat, protein, and carbohydrate, respectively) during 15 weeks. Body weight (BW) gain, terminal BW, and daily energy intake were monitored once weekly along the first 12 weeks, and twice a week during treatment period. The latter was calculated from mean food ingestion per week using the energy density index provided by the manufacturer $(3.34 \mathrm{kcal} / \mathrm{g}$ for $\mathrm{CD}$ or $4.73 \mathrm{kcal} / \mathrm{g}$ for HFD).

In order to assess the potential metabolic effects of $\Delta^{9}$-THCA-A, mice were treated daily by intraperitoneal injection of this compound $(20 \mathrm{mg} / \mathrm{Kg}$ dissolved in ethanol/cremophor/saline

460 1:1:18) for three weeks, from week 12 onwards, in CD and HFD groups ( $\mathrm{n}=10$ /group). The amount of fat mass and adiposity in all set of experiments were measured by Quantitative magnetic resonance (QMR) scans, using the EchoMRI ${ }^{\mathrm{TM}} 700$ analyzer (Houston, TX, USA, software v.2.0), before initiation of the treatments with $\Delta^{9}$-THCA-A and at the end of the experimental procedures. At this point, mice were euthanized and blood and brown adipose tissue (BAT), white adipose tissue (WAT) and liver were collected. Tissues were snap-frozen on dry ice and/or fixed in $4 \%$ formalin for further analysis of molecular expression and histology, respectively.

In another set of experiment, eight-week old male C57BL6 mice fed with CD were treated with $\Delta^{9}$-THCA-A (20 mg/Kg i.p.), with or without the selective PPAR $\gamma$ inhibitor, T0070907

470 (5 mg/Kg i.p.), for 3 weeks ( $\mathrm{n}=10$ /group). Pair-aged animals treated with vehicle served as control ( $\mathrm{n}=10 /$ group). 


\section{Immunohistochemistry and protein analysis by Western Blots.}

Liver and iWAT tissues were fixed in formalin for 24 hours, embedded in paraffin and sectioned. Liver tissue sections $(5 \mu \mathrm{m})$ were stained with haematoxylin and eosin (H\&E). Slides were evaluated for steatosis according to the Kleiner system. A semi-quantitative score was assigned to describe the extent of steatosis $(0,<5 \% ; 1,5-33 \% ; 2,33-66 \%$; and $3,>66 \%)$. IHC analysis of iWAT tissue sections $(7 \mu \mathrm{m})$ was carried out as described previously (48). Briefly, antigen retrieval was performed in trypsin $(\mathrm{pH} 7.8)$ or $10 \mathrm{mM}$ sodium citrate buffer (pH 6) and then incubated with F4/80 antibody (1:50; MCA497, Bio-Rad) or UCP-1 antibody (1:500; ab10983, Abcam, Cambridge, UK) overnight at $4{ }^{\circ} \mathrm{C}$, respectively. Samples were analysed with a Leica DM2000 microscope and pictures were taken with a Leica MC190 camera. For Western blots, proteins were isolated from inguinal white (iWAT) adipose tissues and $30 \mu \mathrm{g}$ of proteins were subjected to SDS/PAGE electrophoresis. Separated proteins were transferred (20V for $30 \mathrm{~min}$ ) to polyvinyl difluoride membranes (PVDF) membranes that were probed probed with antibodies anti-UCP-1 (1:2000, Ab10983, Abcam) and $\alpha$-tubulin (1:10.000; DM-1A, Sigma Aldrich). Differentiated 3T3-L1 cells in adipogenic medium were preincubated with either $\Delta^{9}$-THCA-A or RGZ and treated with $50 \mathrm{ng} / \mathrm{ml} \mathrm{TNF} \alpha$ for $30 \mathrm{~min}$ and the expression of PPAR $\gamma$ Ser272 and total PPAR $\gamma$ analysed with the antibodies anti-PPAR $\gamma$ Ser272 (1:200, bs-4888R, Bioss, Woburn, MA, USA) and anti-PPAR $\gamma$ (D1:1.000, 2435, Cell Signaling Technology, Danvers, MA, USA). Protein levels were normalized to $\beta$-actin (1:5000 dilution, A5060, Sigma Aldrich). Membranes were washed and incubated with the appropriate horseradish peroxidase-conjugated secondary antibody for $1 \mathrm{~h}$ at room temperature and detected by chemiluminescence system (GE Healthcare Europe GmbH, Freiburg, Germany).

\section{Intraperitoneal glucose and insulin tolerance tests, and triglyceride determinations.}

The animals were ip injected with a bolus of $2 \mathrm{~g}$ of glucose per $\mathrm{kg} \mathrm{BW}$, after a $5 \mathrm{~h}$ period of food deprivation, and blood glucose levels were determined at 0, 20, 60 and 120 min after

500 injection. For ITT, the animals were subjected to ip injection of $1 \mathrm{U}$ of insulin (Sigma Aldrich) per kg body weight, after a $5 \mathrm{~h}$ fasting. Blood glucose levels were measured at 0, 20, 60 and 120 minutes. All glucose concentrations were measured using a handheld glucometer (Accu-Check Advantage ${ }^{\circledR}$; Roche Diagnostics, Rotkreuz, Switzerland). In addition, serum triglyceride levels were assayed, using a GPO-POD assay kit (Triglyceride Liquid kit 992320, 


\section{Determination of hormonal, metabolic and inflammatory markers.}

Circulating adipokine levels of Leptin, Insulin, Glucagon, Glucagon-like peptide 1 (GLP-1) and Adiponectin were measured using quantitative Bio-Plex Pro ${ }^{\mathrm{TM}}$ Mouse Diabetes 8-Plex immunoassay (\#171F7001M; Bio-Rad Laboratories, Hercules, CA, USA) and Bio-Plex Pro Mouse Diabetes Adiponectin assay \#171F7002M (Bio-Rad Laboratories, Hercules, CA, USA) according to the manufacturer's instructions. For proteome array, plasma samples from mice were pooled ( $\mathrm{n}=6$ mice per group) and assayed for cytokine and adipokine expression. To study protein expression profiles, $100 \mu \mathrm{l}$ plasma samples were used in the Proteome

515 Profiler Mouse XL Cytokine Array and the Proteome Profiler Mouse Adipokine Array (R\&D Systems, Minneapolis, MN, USA) according to the manufacturer's protocols. Spot density was determined using Quick Spots image analysis software (R\&D Systems).

\section{RNA-Seq and bioinformatic analysis}

520 For each group, total RNA was extracted from iWAT, prepared, pooled, and run on an Agilent Bioanalyzer system to confirm quality (RNA integrity number $>8$ ). Transcriptome libraries were then constructed using poly-A selection with the TruSeq Stranded mRNA Library Prep Kit (Cat. No. RS-122-2101, Illumina, San Diego, CA, USA). In brief, 300 ng of total RNA from each sample was used to construct a cDNA library, followed by sequencing on the Illumina HiSeq 2500 with single end $50 \mathrm{bp}$ reads and $\sim 30$ millions of reads per sample. The FASTQ files were pre-processed with Trimmomatic (v0.36) (52) to remove adapter sequences and aligned to the mm10 assembly of the mouse genome using HISAT2 (v2.1.0) (53). The counts per gene matrix were obtained from the alignments with featureCounts (v1.6.1) (54) using the in-built RefSeq annotation for the mm10 genome assembly. After filtering genes with less than 15 reads across samples, the raw counts were analyzed with DESeq2 (v1.20.0) (55) to obtain the regularized log transformed expression matrix and the differential expression analysis results. We used a threshold of an absolute fold change $\geq 2$ and an adjusted $\mathrm{P}$ value $\leq 0.01$ to consider a gene as differentially expressed in any comparison. Heatmaps were generated using the scaled mean of the regularized log expression for each group with the $\mathrm{R}$ package ComplexHeatmap (v1.20.0). The gene set enrichment analysis (GSEA) (56) and the over-representation analysis were performed using the R package ClusterProfiler (v3.10.1) (57). For GSEA, genes were pre-ranked using the $\log 2$ transformed fold change. The KEGG pathway database and Gene Ontology (Biological Process) annotation were used to group genes by biological function. All the $\mathrm{P}$ values were 
540 adjusted using the Benjamini and Hochberg correction to control the false discovery rate (FDR).

\section{Real-time PCR.}

Total RNA was isolated at day 7 or 14 of MSCs differentiation using the High Pure RNA 545 Isolation kit (Roche Diagnostics). For tissues, total RNA was isolated using the Qiagen RNeasy Lipid Kit (Qiagen, Hilden, Germany). For real-time PCR analysis, RNA was reverse transcribed to cDNA using the iScript ${ }^{\mathrm{TM}}$ cDNA Synthesis Kit (Bio-Rad), and the cDNA generated was analysed by real-time PCR using the iQTM SYBR Green Supermix (Bio-Rad) using a CFX96 Real-Time PCR Detection System (Bio-Rad). Gene expression was normalized to HPRT or GADPH mRNA levels in each sample. The HPRT or GAPDH gene was used to standardize mRNA expression in each sample. The primers used are listed in Supplementary Table S2.

\section{Statistical analysis.}

555 In vitro data are expressed as mean \pm SD with a minimal of 3 to 4 independent experiments. In vivo results are represented as mean \pm SEM and the determinations were conducted with a minimal total number of 6-10 animals per group. Statistical analyses were performed on data distributed in a normal pattern, using Student's t tests or ANOVA followed by Tukey's test. P $<0.05$ was taken as the minimum level of significance. Statistical analysis was performed using GraphPad Prism ${ }^{\circledR}$ version 6.01 . 


\section{AUTHOR CONTRIBUTIONS}

$\mathrm{BP}, \mathrm{RM}$ and MEP conducted the in vitro experiments, the histopathology and the immunohistochemistry studies. FRP coordinated and conducted, together with BP, IV, MASG, MEP and $\mathrm{MJV}$, the whole set of in vivo experiments, as well as different of the analytical procedures related with those. MGR and MAC conducted the in-silico experiments and the bioinformatic analysis. BP, FRP, MAC and MGR were responsible for initial data analysis, figure preparation and statistical analysis. XN and CFV provided several batches of $\Delta^{9}$-THCA-A. MTS, GM and GA had a leading contribution in the design of in vivo studies, and an active role in the discussion and interpretation of the whole dataset. EM was responsible for the overall design of study, and major coordinator of the whole set of experiments. EM and MTS jointly wrote the manuscript as well as by the rest of the authors. All the authors take full responsibility for the work.

\section{ACKNOWLEDGEMENTS}

575 This work was supported by grants SAF2017-87701-R (EM) and BFU2014-57581-P and BFU2017-83934-P (MTS) (Ministerio de Economía y Competitividad, Spain; co-funded with EU funds from FEDER Program); Project P12-FQM-01943 (M.T.-S.; Junta de Andalucía, Spain). CIBER Fisiopatología de la Obesidad y Nutrición is an initiative of Instituto de Salud Carlos III. None of the funding bodies played any role in the study design, data collection and analysis,

580 the decision to publish, or the preparation of the manuscript. BP is a predoctoral fellow supported by the i-PFIS program, Instituto de Salud Carlos III (IFI15/00022; European Social Fund "investing in your future").

\section{CONFLICT OF INTEREST}

585 The authors declare no conflict of interest in relation to the contents of this work. 


\section{FIGURE LEGENDS}

Figure 1. Characterization of $\boldsymbol{\Delta}^{\mathbf{9}}$-THCA-A as a selective PPAR $\gamma$ agonist. (A) Receptorspecific transactivation by $\Delta^{9}$-THCA-A. HEK-293T cells were co-transfected with the plasmids encoding nuclear receptors (GAL4-PPAR $\gamma$, GAL4- PPAR $\alpha$ and GAL4-PPAR $\delta$ ) and their cognate luciferase reporter (GAL4-luc). After transfection, cells were treated with $\Delta^{9}$ THCA-A $(1 \mu \mathrm{M})$ and receptor-specific agonists for 6 hours. Control (black bars), VCE-004.8 (blue bars) and specific ligands for each receptor (grey bars): RGZ $(1 \mu \mathrm{M})$ for PPAR $\gamma$, WY14643 $(5 \mu \mathrm{M})$ for PPAR $\alpha$, and GW0742 $(5 \mu \mathrm{M})$ for PPAR $\delta$. Results are expressed as the fold induction \pm SD $(n=3)$ relative to untreated control. (B) Representative Western blot images of PPAR $\gamma$ phosphorylation at Ser273 in 3T3L1 adipocytes pre-treated with $\Delta^{9}$-THCAA and RGZ for $30 \mathrm{~min}$, followed by treatment with TNF- $\alpha$ for $30 \mathrm{~min}(\mathrm{n}=3)$. (C, D) HEK293T cells were co-transfected with GAL4-PPAR $\gamma$ and GAL4-luc, pre-treated with T0070907 for $15 \mathrm{~min}$ and then stimulated with increasing concentrations of either RGZ or $\Delta^{9}$-THCA-A 605 for 6 hours and assayed for luciferase activity. Results are expressed as the fold induction \pm SD relative to RGZ (C) or $\Delta^{9}$-THCA-A (D) $(\mathrm{n}=4)$. (E, F) Adipocyte differentiation of MSCs in adipogenic medium with RGZ (E) or $\Delta^{9}$-THCA-A (F) in the presence and the absence of T0070907 for 7 days. mRNA levels of adipogenic markers were analysed by qPCR. Results represent the mean $\pm \mathrm{S} . \mathrm{D}(\mathrm{n}=3)$. ${ }^{*} \mathrm{P}<0.05, * * \mathrm{P}<0.01$ and $* * * \mathrm{P}<0.001$ agonist ligands or $\Delta^{9}$-THCA-A treatment vs. control; RGZ + T0090709 vs. RGZ; RGZ or $\Delta^{9}$-THCA-A treatment vs. control; ${ }^{\# \#} \mathrm{P}<0.01$ and ${ }^{\# \# \#} \mathrm{P}<0.001 \mathrm{RGZ}+\mathrm{T} 0090709$ vs RGZ or $\Delta^{9}-\mathrm{THCA}-\mathrm{A}+$ T0090709 vs $\Delta^{9}$-THCA-A. (ANOVA followed by Tukey's test or unpaired two-tailed Student's t-test).

Figure 2. Transcriptomic analysis of the PPAR $\gamma$ dependent activity of $\Delta^{9}$-THCA-A in iWAT. (A) Heatmap of all the differentially expressed genes (absolute fold change $\geq 2$ and an adjusted $\mathrm{P}$ value $\leq 0.01)$ in $\Delta^{9}$-THCA-A versus control or $\Delta^{9}$-THCA-A+T0070907 versus control comparisons. The color represents the scaled mean of log transformed expression. The column annotations indicate the sample group and the points at the right side highlight the position of genes belonging to the KEGG pathways of interest. (B) Gene set enrichment analysis results for the KEGG pathways of interest. The left side enrich plots indicate the position of the genes belonging to each pathway in the pre-ranked list per comparison. The right-side bar plots represent the normalized enrichment score (NES) and significance of the GSEA result $* \mathrm{P} \leq 0.05 ; * * \mathrm{P} \leq 0.01 ; * * * \mathrm{P} \leq 0.001$. (C) Pie charts indicating the number and 
proportion of differentially expressed genes included in the KEGG pathways of interest for each comparison. (D) Heatmap of the top 25 genes induced by $\Delta^{9}$-THCA-A inside the PPAR signaling or thermogenesis pathways that are not differentially expressed in the $\Delta^{9}$-THCAA+T0070907 vs control comparison. (E) Representative Western blot images of UCP-1 protein expression and immuno-histochemistry with anti-UCP-1 antibodies in iWAT tissue (original magnification $\times 10$, scale bar: $200 \mu \mathrm{m})(\mathrm{n}=3$ ).

\section{Figure 3. Effect of $\Delta^{9}$-THCA-A on MSCs adipogenic and osteoblastogenic differentiation.}

MSCs were cultured under adipogenic medium (AM) in the presence of RGZ or $\Delta^{9}$-THCA-A. (A) Representative images of the cells stained with Oil Red O assayed by light microscopy

$635(\times 10)$ after 21 days of differentiation. (B) Quantification of the stained lipid droplets were performed measuring absorbance at 540nm. (C) mRNA levels of adipogenic markers were analysed by qPCR after 14 days of differentiation. MSCs were cultured under osteoblastogenic medium (OM) in the presence of $\Delta^{9}$-THCA-A. (D) Mineralization detected by Alizarin red staining was assessed by gross appearance after 21 days of

640 differentiation. (E) Quantification of the eluted Alizarin Red stain measuring absorbance at $405 \mathrm{~nm}$. (F) Gene expression of osteoblastogenic markers analysed by qPCR. Results represent the mean $\pm \mathrm{S} . \mathrm{D}(\mathrm{n}=3) . * \mathrm{P}<0.05, * * \mathrm{p}<0.01$ and $* * * \mathrm{P}<0.001 \mathrm{RGZ}$ or $\Delta^{9}-\mathrm{THCA}-$ A vs. AM; $\Delta^{9}$-THCA-A vs. OM. (ANOVA followed by Tukey’s test).

645 Figure 4. Effect of administration of $\Delta^{9}$-THCA-A on metabolic and hormonal parameters in a mouse model of HFD-induced obesity. (A) Body weight (BW) evolution of adult male mice fed for 12-weeks with high fat diet (HFD) or the corresponding control diet (CD). (B) BW change in HFD and CD mice treated for three weeks with $\Delta^{9}$-THCA-A or vehicle; values are referenced to $\mathrm{BW}$ at the beginning of treatment (taken as 0). (C)

650 Percentage of adiposity, at the end of treatments in the four experimental groups. (D-E) Glucose and Insulin tolerance tests in CD and HFD mice treated with $\Delta^{9}$-THCA-A or vehicle for three weeks. (F) Basal insulin levels at the end of the three-week treatment period are shown for the four experimental groups. (G) Liver sections with hematoxylin and eosin (H\&E) staining (original magnification x10, scale bar: $200 \mu \mathrm{m})$. (H) Steatosis scores $(\mathrm{n}=6$

655 mice per group) and (I) plasma levels of triglycerides. Values correspond to means $\pm \mathrm{SEM}$ of at least 8 mice per group. ${ }^{*} P<0.05,{ }^{* *} P<0.01,{ }^{* * *} P<0.001 \Delta^{9}$-THCA-A-treated mice or HFD mice vs. control (CD) mice; ${ }^{\#} P<0.05,{ }^{\#} P<0.01,{ }^{\# \#} P<0.001 \quad \Delta^{9}$-THCA-A-treated HFD mice 
vs. HFD mice treated with vehicle (ANOVA followed by Tukey's test or unpaired two-tailed Student's t-test).

660

Figure 5. Transcriptomic analysis of $\Delta^{\mathbf{9}}$-THCA-A effects in the iWAT of HFD mice. (A) Heatmap of all the differentially expressed genes (absolute fold change $\geq 2$ and an adjusted $\mathrm{P}$ value $\leq 0.01)$ in HFD versus control or HFD $+\Delta^{9}-$ THCA-A versus control comparisons. The color represents the scaled mean of log transformed expression. The column annotations indicate the sample group and the points at the right side highlight the position of genes belonging to the KEGG pathways of interest. (B) Gene set enrichment analysis results for the KEGG pathways of interest. The left side enrich plots indicate the position of the genes belonging to each pathway in the pre-ranked list per comparison. The right-side bar plots represent the normalized enrichment score (NES) and significance of the GSEA result $* \mathrm{P} \leq$ $0.05 ; * * \mathrm{P} \leq 0.01 ; * * * \mathrm{P} \leq 0.001$. (C) Pie charts indicating the number and proportion of differentially expressed genes included in the KEGG pathways of interest for each comparison. (D) Heatmap of the top 25 genes induced by HFD inside the NF- $\mathrm{KB}$ or cytokinecytokine receptor pathways that are not differentially expressed in the HFD $+\Delta^{9}-\mathrm{THCA}-\mathrm{A}$ vs control comparison. (E) Gene expression of pro-inflammatory genes were measured by 675 qPCR. Results are presented as mean \pm SEM of at least 5 mice per group. ${ }^{*} \mathrm{P}<0.05, * * \mathrm{P}<0.01$ HFD mice vs. control mice; \#P<0.05, \#\#P $<0.01 \quad \Delta 9$-THCA-A-treated HFD mice vs. HFD mice treated with vehicle (ANOVA followed by Tukey's test).

Figure 6. Effects of $\Delta^{9}$-THCA-A on iWAT browning, adiposity and circulating factors, with key roles in metabolic homeostasis in CD and HFD animals. (A) Crown Like Structures (CLS) and browning in iWAT. Representative immunohistochemical detection of anti-F4/80 and anti-UCP-1 antibodies (original magnification x20, scale bar: $100 \mu \mathrm{m}$ ), (B) Quantification of adipocyte area ( $\mathrm{n}=6$ animals per group), (C) UCP-1 protein levels determined by western blotting in iWAT tissue $(n=3)$. Hormonal markers linked to energy and metabolic homeostasis assayed: (D) Leptin, (E) GLP-1, (F) Glucagon and (G) Adiponectin. (I) Heatmap showing the plasma profile of cytokines and adipokines. Values correspond to means \pm SEM of at least 8 mice per group. ${ }^{*} P<0.05,{ }^{* * *} P<0.001$ HFD mice vs. control (CD) mice; ${ }^{\#} P<0.05,{ }^{\# \# \#} P<0.001 \Delta^{9}$-THCA-A-treated HFD mice vs. HFD mice treated with vehicle (ANOVA followed by Tukey's test or unpaired two-tailed Student's t-test). 


\section{References}

1. Hanus LO, Meyer SM, Munoz E, Taglialatela-Scafati O, \& Appendino G (2016) Phytocannabinoids: a unified critical inventory. Natural product reports 33(12):13571392.

2. Krejci Z, Horak M, \& Santavy F (1959) [Hemp (Cannabis sativa)-an antibiotic drug. 3. Isolation and constitution of two acids from Cannabis sativa]. Die Pharmazie 14:349-355.

700 3. Wang M, et al. (2016) Decarboxylation Study of Acidic Cannabinoids: A Novel Approach Using Ultra-High-Performance Supercritical Fluid Chromatography/Photodiode Array-Mass Spectrometry. Cannabis and cannabinoid research 1(1):262-271.

4. Moreno-Sanz G (2016) Can You Pass the Acid Test? Critical Review and Novel Therapeutic Perspectives of Delta(9)-Tetrahydrocannabinolic Acid A. Cannabis and cannabinoid research 1(1):124-130.

5. Nadal X, et al. (2017) Tetrahydrocannabinolic acid is a potent PPARgamma agonist with neuroprotective activity. British journal of pharmacology 174(23):4263-4276.

6. Bluher M (2019) Obesity: global epidemiology and pathogenesis. Nature reviews. Endocrinology 15(5):288-298.

7. Kaur J (2014) A comprehensive review on metabolic syndrome. Cardiology research and practice 2014:943162.

8. Bray GA, et al. (2018) The Science of Obesity Management: An Endocrine Society Scientific Statement. Endocrine reviews 39(2):79-132.

7159 9. Anonymous (2014) Guidelines (2013) for managing overweight and obesity in adults. Preface to the Expert Panel Report (comprehensive version which includes systematic evidence review, evidence statements, and recommendations). Obesity 22 Suppl 2:S40.

10. Bhaskaran K, et al. (2014) Body-mass index and risk of 22 specific cancers: a

population-based cohort study of 5.24 million UK adults. Lancet 384(9945):755-765.

11. Karczewski J, et al. (2018) Obesity and inflammation. European cytokine network 29(3):83-94.

12. Forny-Germano L, De Felice FG, \& Vieira M (2018) The Role of Leptin and Adiponectin in Obesity-Associated Cognitive Decline and Alzheimer's Disease. Frontiers in neuroscience 12:1027.

13. Schwartz MW, et al. (2017) Obesity Pathogenesis: An Endocrine Society Scientific Statement. Endocrine reviews 38(4):267-296.

14. Wang T \& He C (2018) Pro-inflammatory cytokines: The link between obesity and osteoarthritis. Cytokine \& growth factor reviews 44:38-50.

730 15. Pirola L \& Ferraz JC (2017) Role of pro- and anti-inflammatory phenomena in the physiopathology of type 2 diabetes and obesity. World journal of biological chemistry 8(2):120-128.

16. Mishra AK, Dubey V, \& Ghosh AR (2016) Obesity: An overview of possible role(s) of gut hormones, lipid sensing and gut microbiota. Metabolism: clinical and experimental 65(1):48-65.

17. Montgomery MK, De Nardo W, \& Watt MJ (2019) Impact of Lipotoxicity on Tissue "Cross Talk" and Metabolic Regulation. Physiology 34(2):134-149.

18. Tschop MH, et al. (2016) Unimolecular Polypharmacy for Treatment of Diabetes and Obesity. Cell metabolism 24(1):51-62. 
740 19. Muller TD, Clemmensen C, Finan B, DiMarchi RD, \& Tschop MH (2018) AntiObesity Therapy: from Rainbow Pills to Polyagonists. Pharmacological reviews 70(4):712-746.

20. Clemmensen C, et al. (2019) Emerging hormonal-based combination pharmacotherapies for the treatment of metabolic diseases. Nature reviews. Endocrinology 15(2):90-104.

21. Loh RKC, Kingwell BA, \& Carey AL (2017) Human brown adipose tissue as a target for obesity management; beyond cold-induced thermogenesis. Obesity reviews : an official journal of the International Association for the Study of Obesity 18(11):12271242.

750 22. Liu J, Wang Y, \& Lin L (2019) Small molecules for fat combustion: targeting obesity. Acta pharmaceutica Sinica. B 9(2):220-236.

23. Lopez M, Nogueiras R, Tena-Sempere M, \& Dieguez C (2016) Hypothalamic AMPK: a canonical regulator of whole-body energy balance. Nature reviews. Endocrinology 12(7):421-432.

755 24. Giralt M \& Villarroya F (2013) White, brown, beige/brite: different adipose cells for different functions? Endocrinology 154(9):2992-3000.

25. Ikeda K, Maretich P, \& Kajimura S (2018) The Common and Distinct Features of Brown and Beige Adipocytes. Trends in endocrinology and metabolism: TEM 29(3):191-200.

760 26. Lopez M \& Tena-Sempere M (2017) Estradiol effects on hypothalamic AMPK and BAT thermogenesis: A gateway for obesity treatment? Pharmacology \& therapeutics 178:109-122.

27. Ramseyer VD \& Granneman JG (2016) Adrenergic regulation of cellular plasticity in brown, beige/brite and white adipose tissues. Adipocyte 5(2):119-129.

765 28. Tontonoz P \& Spiegelman BM (2008) Fat and beyond: the diverse biology of PPARgamma. Annual review of biochemistry 77:289-312.

29. Wang H, Liu L, Lin JZ, Aprahamian TR, \& Farmer SR (2016) Browning of White Adipose Tissue with Roscovitine Induces a Distinct Population of UCP1(+) Adipocytes. Cell metabolism 24(6):835-847.

770 30. Ahmadian M, et al. (2013) PPARgamma signaling and metabolism: the good, the bad and the future. Nature medicine 19(5):557-566.

31. Weidner C, et al. (2012) Amorfrutins are potent antidiabetic dietary natural products. Proceedings of the National Academy of Sciences of the United States of America 109(19):7257-7262.

775 32. Chrisman IM, et al. (2018) Defining a conformational ensemble that directs activation of PPARgamma. Nature communications 9(1):1794.

33. Hughes TS, et al. (2012) Ligand and receptor dynamics contribute to the mechanism of graded PPARgamma agonism. Structure 20(1):139-150.

34. Hughes TS, et al. (2014) An alternate binding site for PPARgamma ligands. Nature $780 \quad$ communications 5:3571.

35. Choi JH, et al. (2011) Antidiabetic actions of a non-agonist PPARgamma ligand blocking Cdk5-mediated phosphorylation. Nature 477(7365):477-481.

36. Gregor MF \& Hotamisligil GS (2011) Inflammatory mechanisms in obesity. Annual review of immunology 29:415-445.

785 37. Hruby A \& Hu FB (2015) The Epidemiology of Obesity: A Big Picture. PharmacoEconomics 33(7):673-689.

38. Amato AA \& de Assis Rocha Neves F (2012) Idealized PPARgamma-Based Therapies: Lessons from Bench and Bedside. PPAR research 2012:978687. 
39. Tan CK, Zhuang Y, \& Wahli W (2017) Synthetic and natural Peroxisome Proliferatorsyndrome. Expert opinion on therapeutic targets 21(3):333-348.

40. Shang J, et al. (2018) Cooperative cobinding of synthetic and natural ligands to the nuclear receptor PPARgamma. eLife 7.

41. Ostberg T, et al. (2004) A new class of peroxisome proliferator-activated receptor agonists with a novel binding epitope shows antidiabetic effects. The Journal of biological chemistry 279(39):41124-41130.

42. Kintscher U \& Goebel M (2009) INT-131, a PPARgamma agonist for the treatment of type 2 diabetes. Current opinion in investigational drugs 10(4):381-387.

43. Marciano DP, et al. (2015) Pharmacological repression of PPARgamma promotes osteogenesis. Nature communications 6:7443.

44. Chung KJ, Nati M, Chavakis T, \& Chatzigeorgiou A (2018) Innate immune cells in the adipose tissue. Reviews in endocrine \& metabolic disorders 19(4):283-292.

45. Zhao Y, et al. (2018) CD4(+) T cells in obesity and obesity-associated diseases. Cellular immunology 332:1-6.

805 46. Reilly SM \& Saltiel AR (2017) Adapting to obesity with adipose tissue inflammation. Nature reviews. Endocrinology 13(11):633-643.

47. Zhang X \& Young HA (2002) PPAR and immune system--what do we know? International immunopharmacology 2(8):1029-1044.

48. Palomares B, et al. (2018) VCE-004.8, A Multitarget Cannabinoquinone, Attenuates Adipogenesis and Prevents Diet-Induced Obesity. Scientific reports 8(1):16092.

49. Di Marzo V, et al. (2001) Leptin-regulated endocannabinoids are involved in maintaining food intake. Nature 410(6830):822-825.

50. Cota D, et al. (2003) The endogenous cannabinoid system affects energy balance via central orexigenic drive and peripheral lipogenesis. The Journal of clinical investigation 112(3):423-431.

51. Verty AN, McGregor IS, \& Mallet PE (2005) Paraventricular hypothalamic CB(1) cannabinoid receptors are involved in the feeding stimulatory effects of Delta(9)tetrahydrocannabinol. Neuropharmacology 49(8):1101-1109.

52. Bolger AM, Lohse M, \& Usadel B (2014) Trimmomatic: a flexible trimmer for

53. Kim D, Langmead B, \& Salzberg SL (2015) HISAT: a fast spliced aligner with low memory requirements. Nature methods 12(4):357-360.

54. Liao Y, Smyth GK, \& Shi W (2014) featureCounts: an efficient general purpose program for assigning sequence reads to genomic features. Bioinformatics 30(7):923930.

55. Love MI, Huber W, \& Anders S (2014) Moderated estimation of fold change and dispersion for RNA-seq data with DESeq2. Genome biology 15(12):550.

56. Subramanian A, et al. (2005) Gene set enrichment analysis: a knowledge-based approach for interpreting genome-wide expression profiles. Proceedings of the National Academy of Sciences of the United States of America 102(43):15545-15550.

57. Yu G, Wang LG, Han Y, \& He QY (2012) clusterProfiler: an R package for comparing biological themes among gene clusters. Omics : a journal of integrative biology 16(5):284-287. 
A

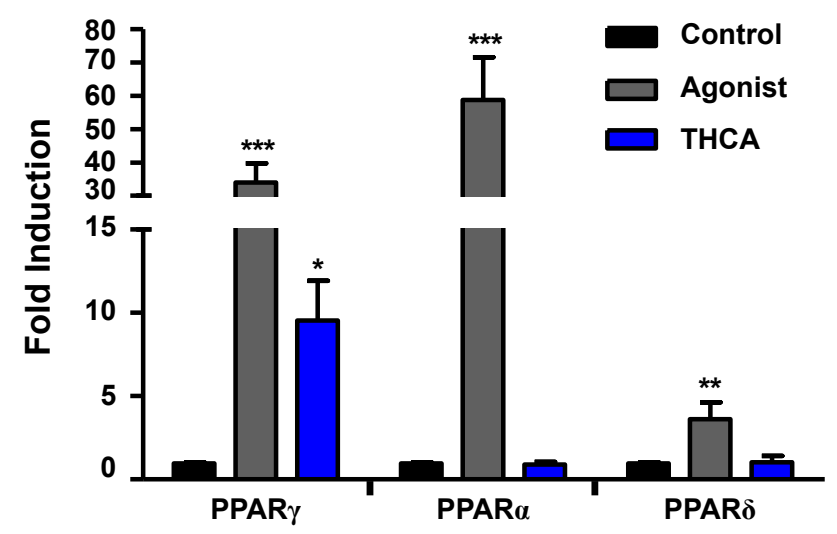

C

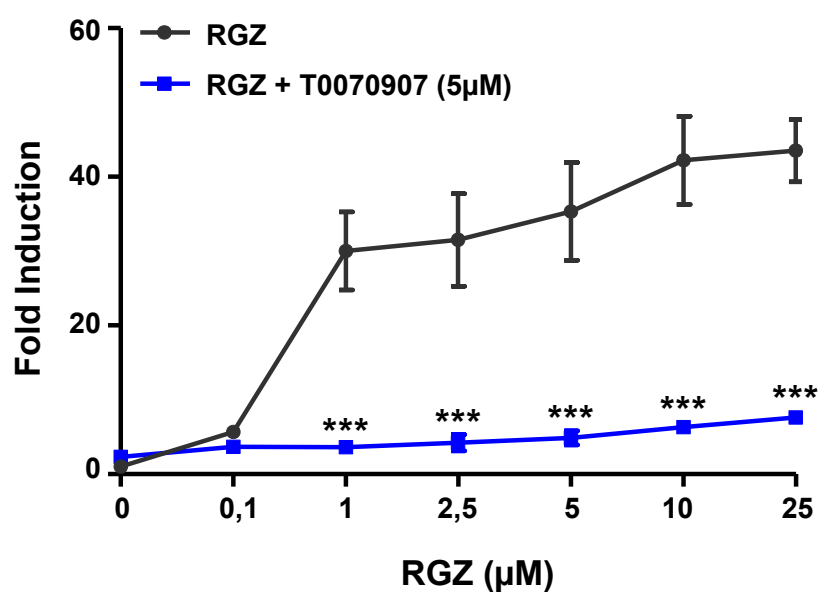

E

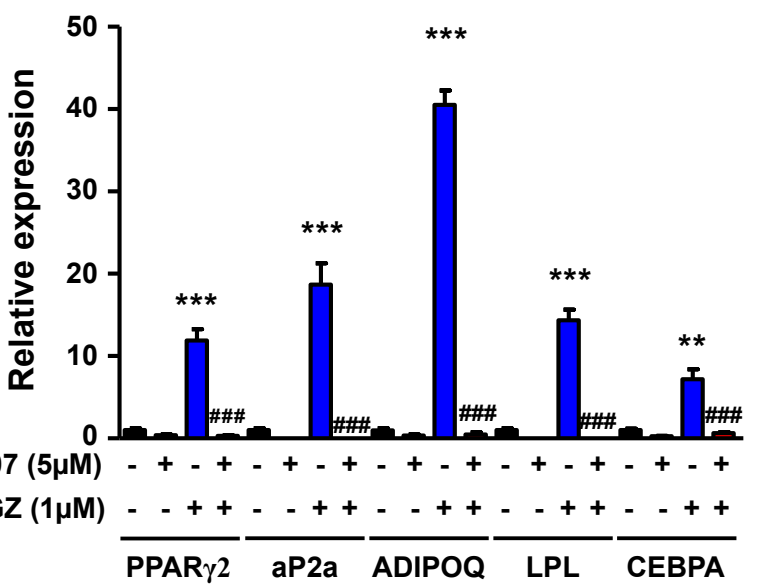

B
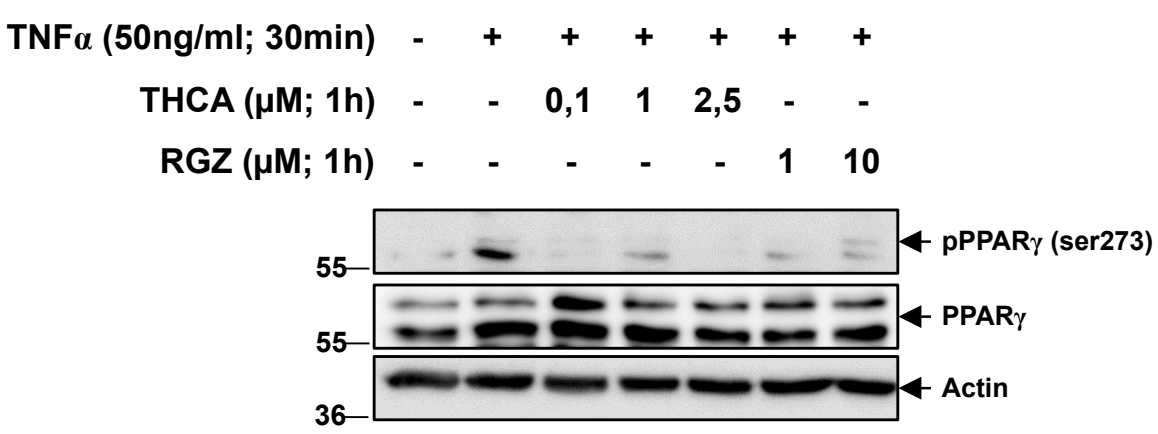

D

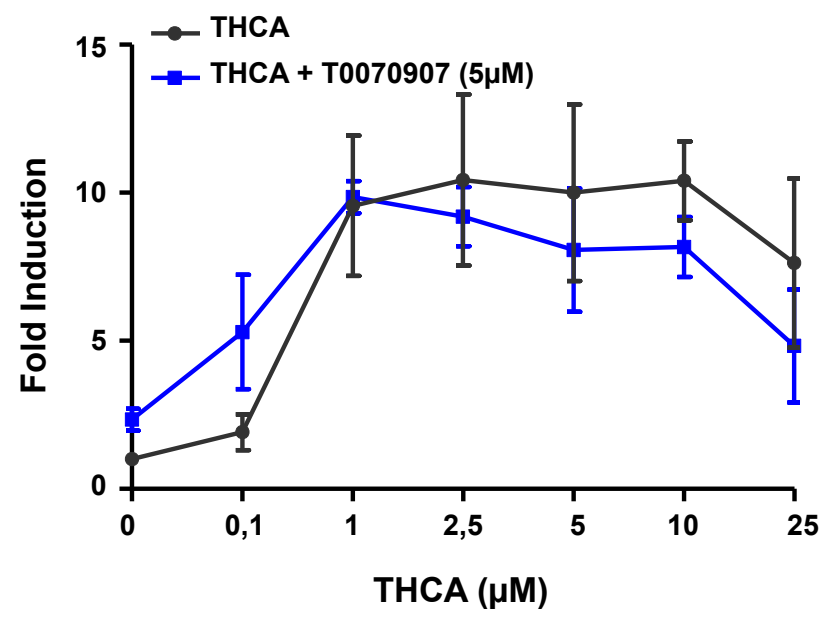

$\mathbf{F}$

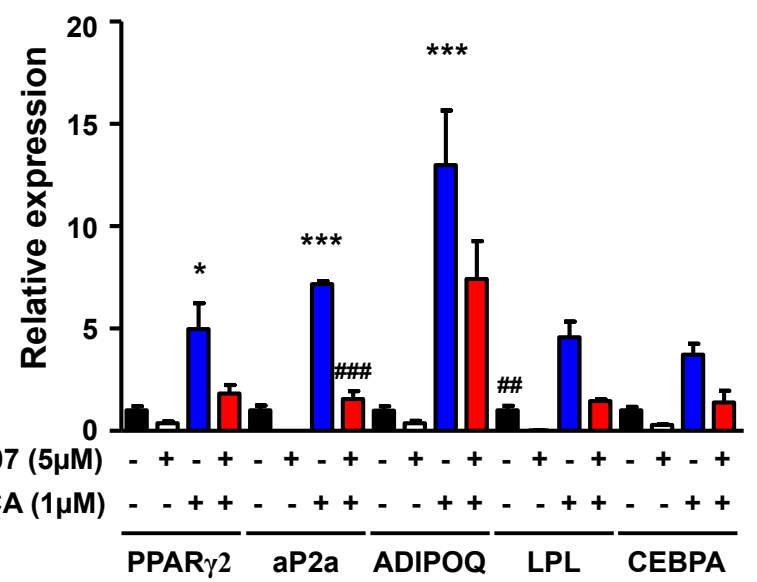


A

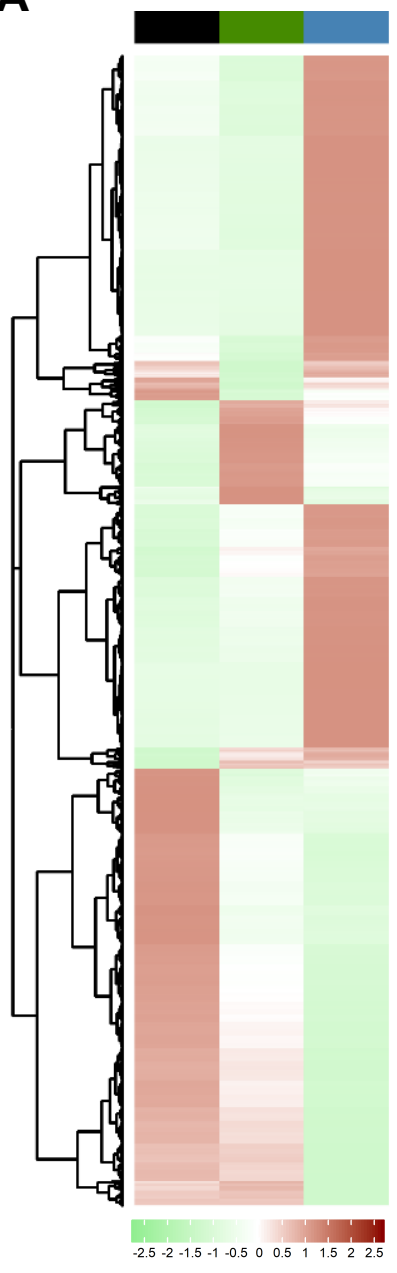

C
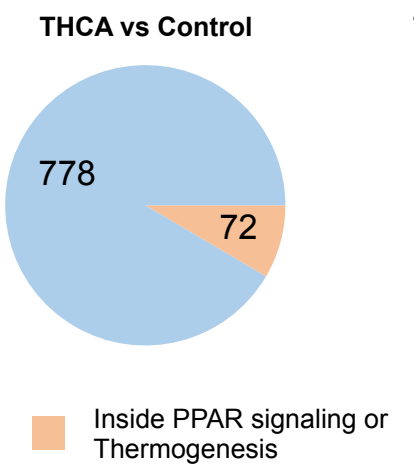

E

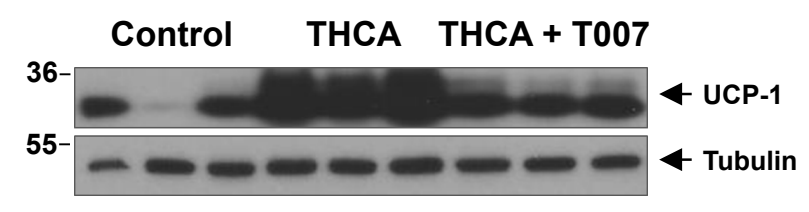

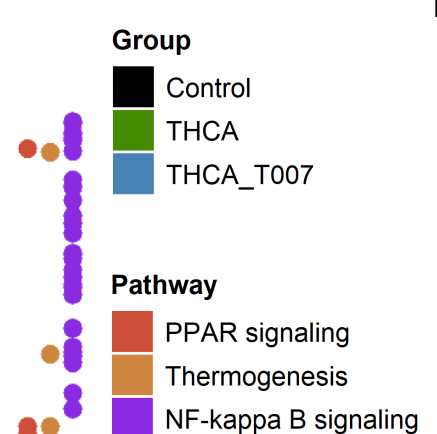

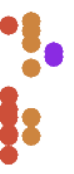

THCA+T007 vs Control

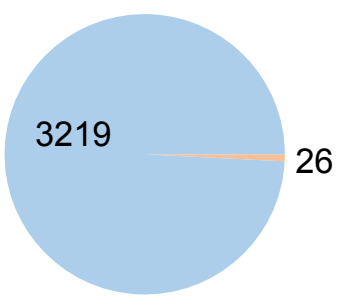

Rest of DEGs
B

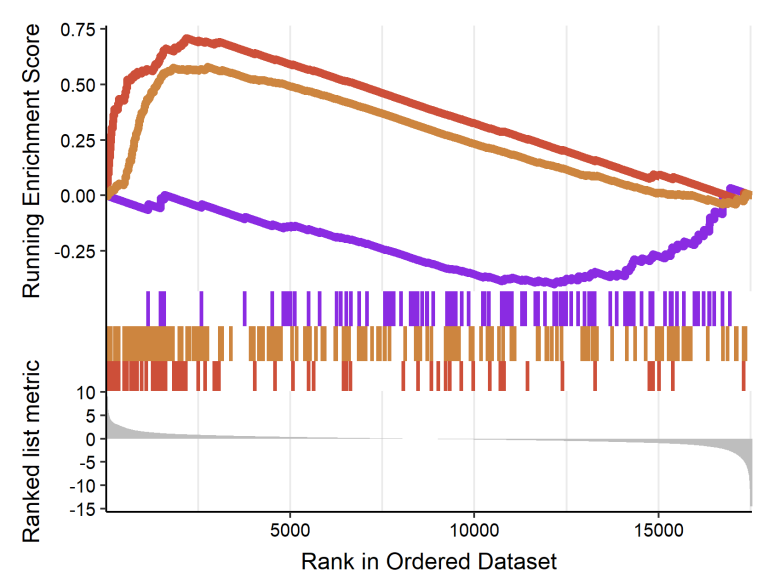

THCA+T007 vs Control

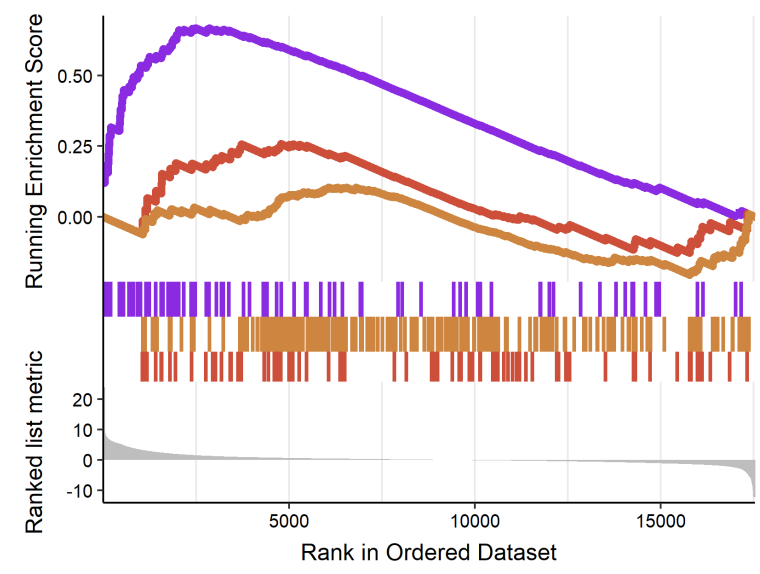

Pathway PPAR signaling Thermogenesis
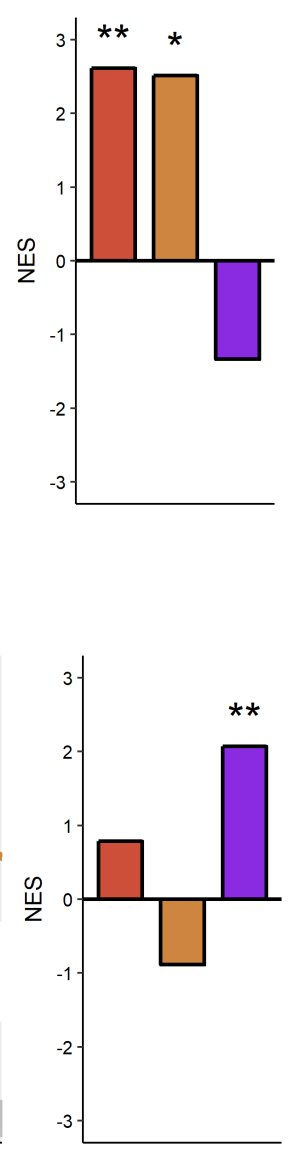

D

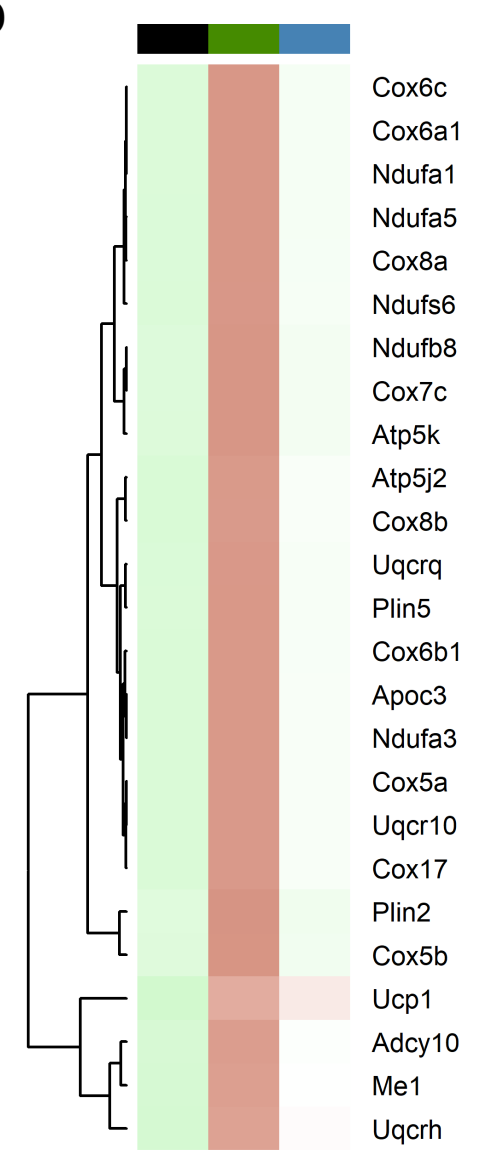


A

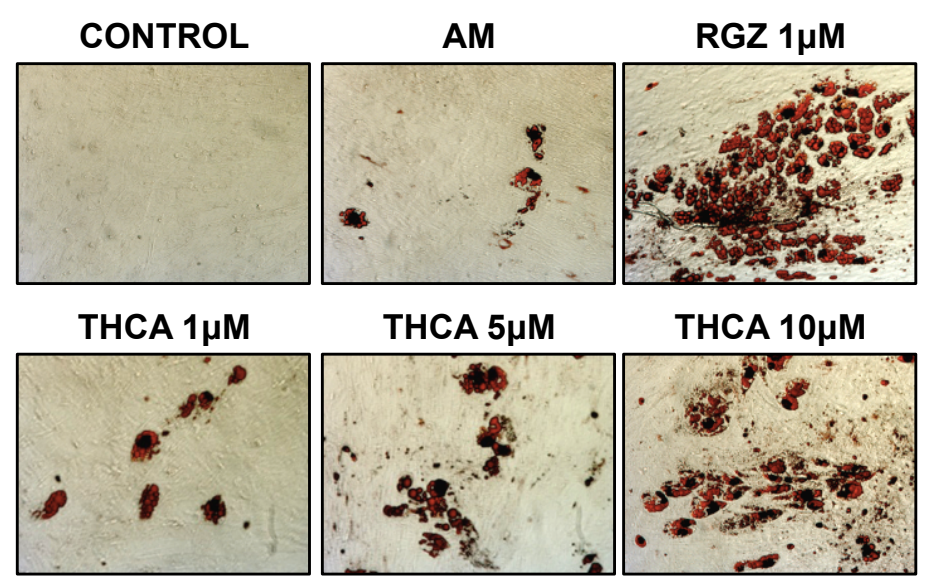

B

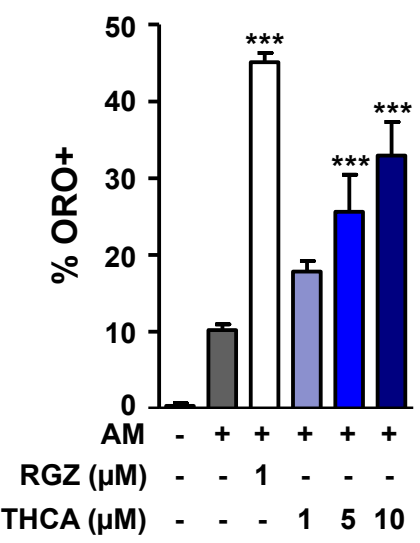

C

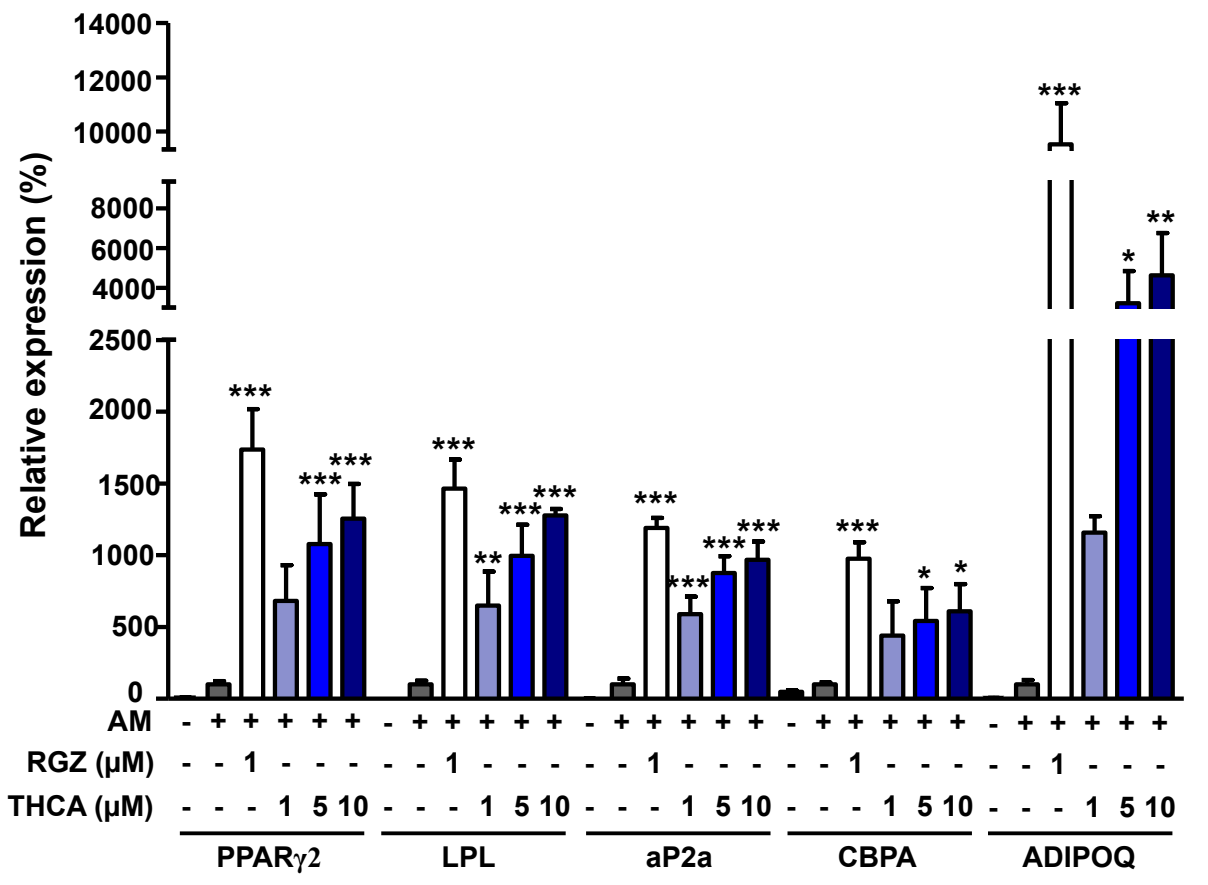

D

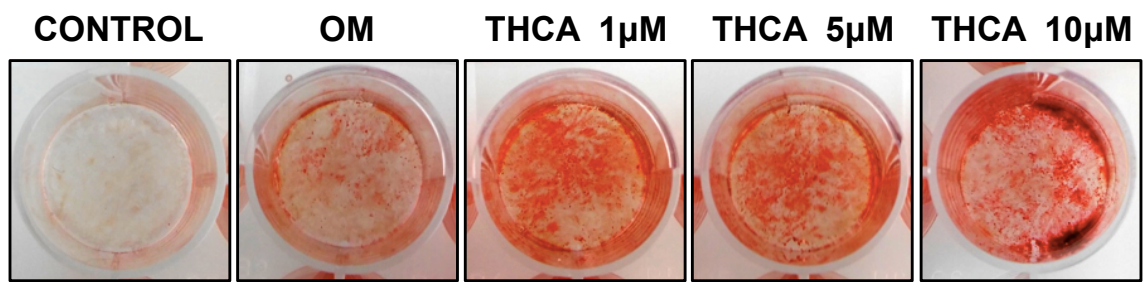

$\mathbf{F}$

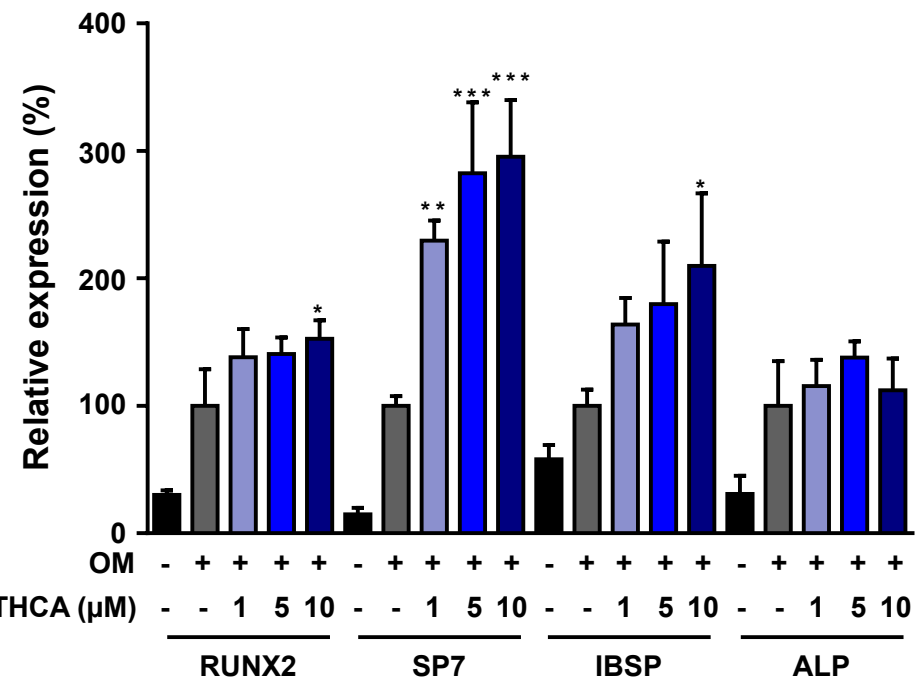

E

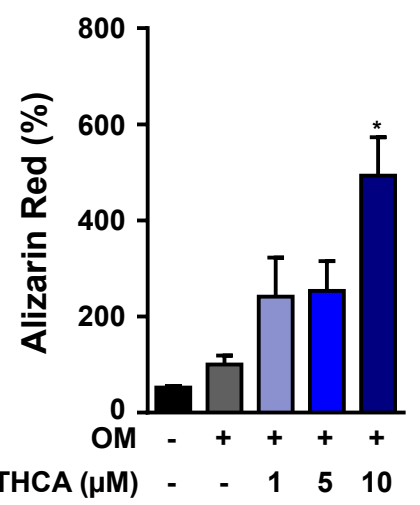


A

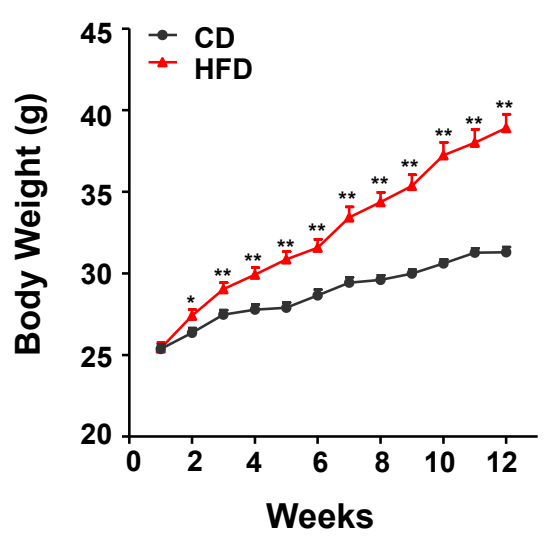

D

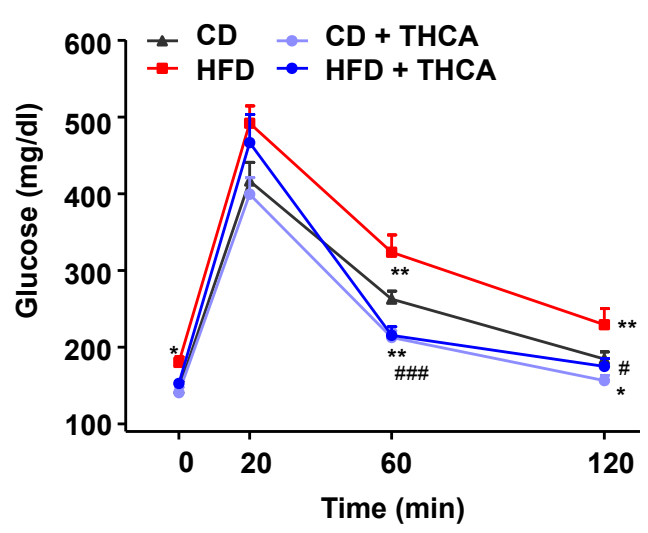

G

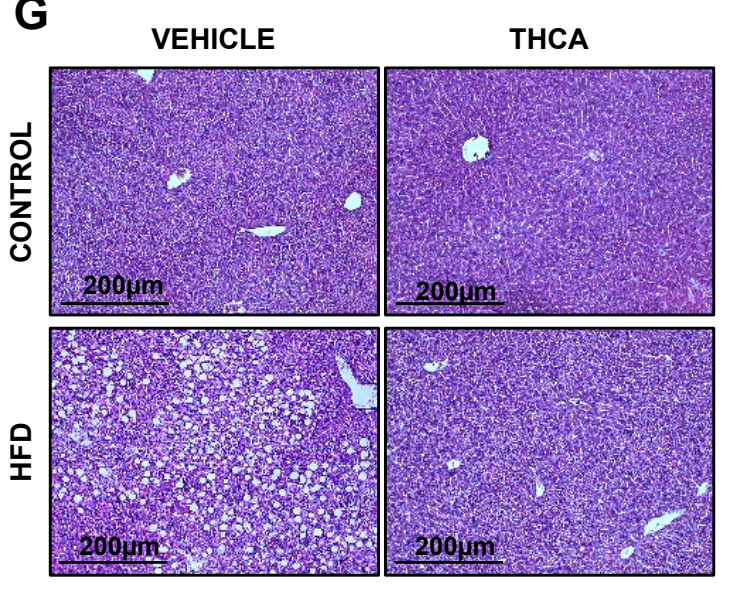

B

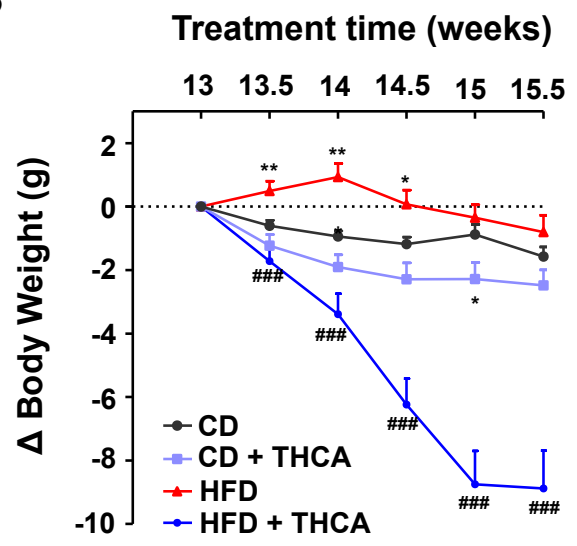

E

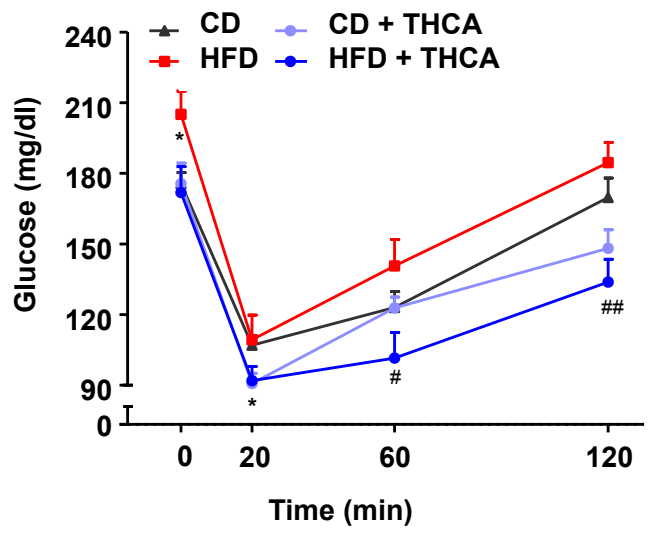

H

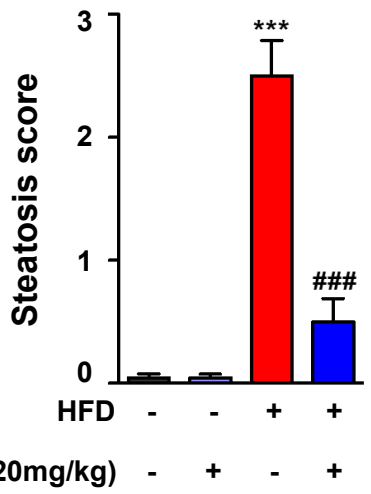

\section{C}

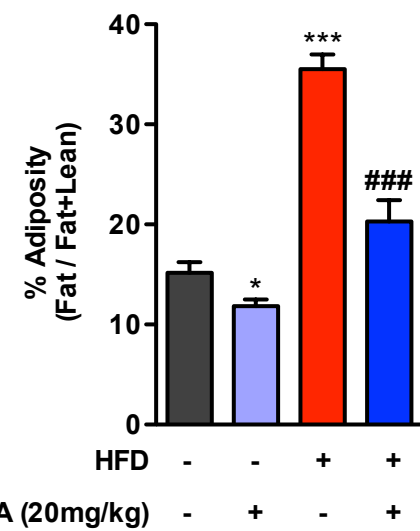

F

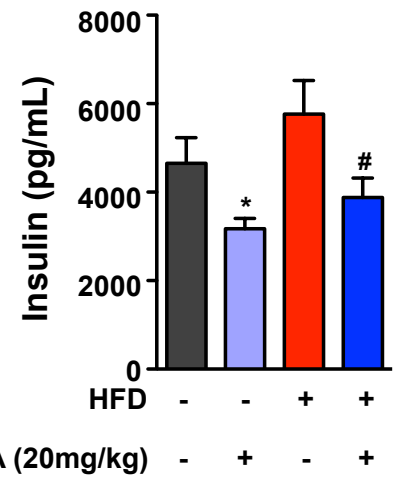

I

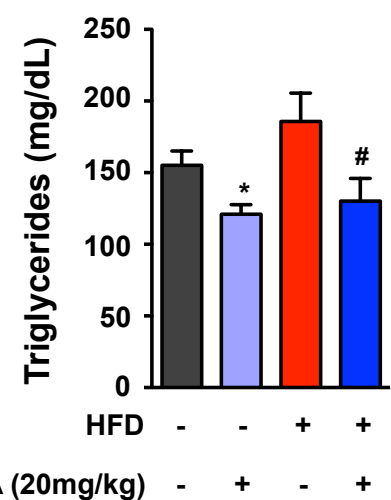


A

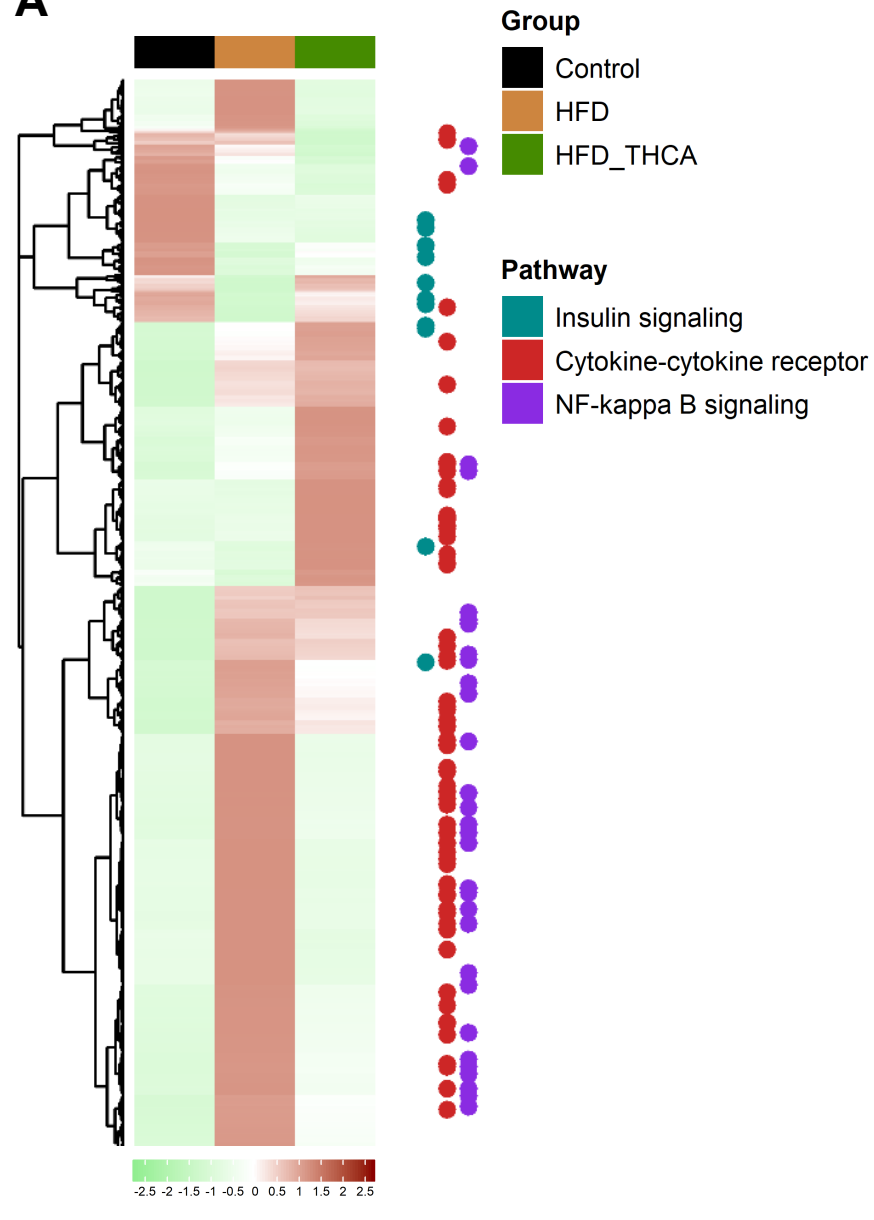

C

HFD vs Control

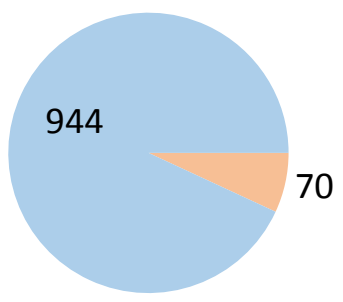

Inside NF-kappa B signaling or

Cytokine-Cytokine receptor

Rest of DEGs

E

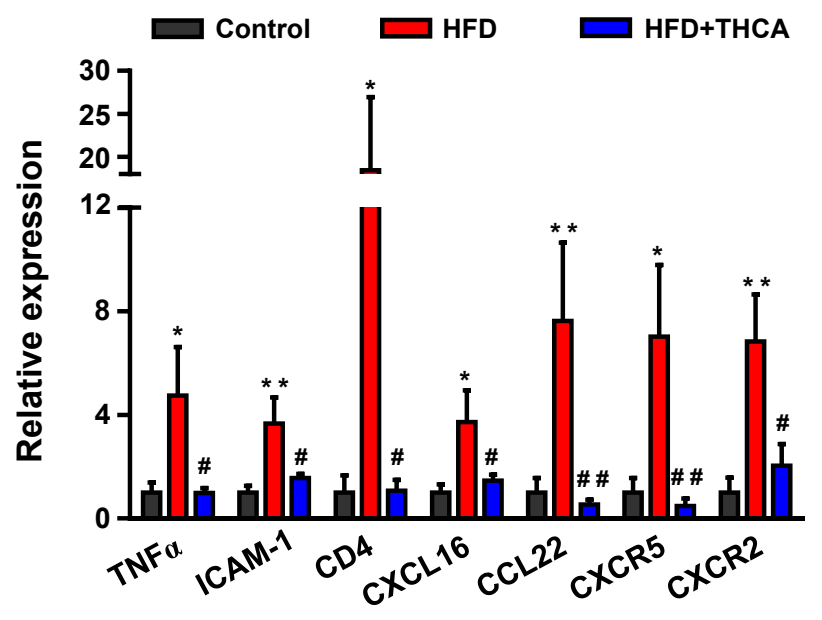

B

HFD vs Control

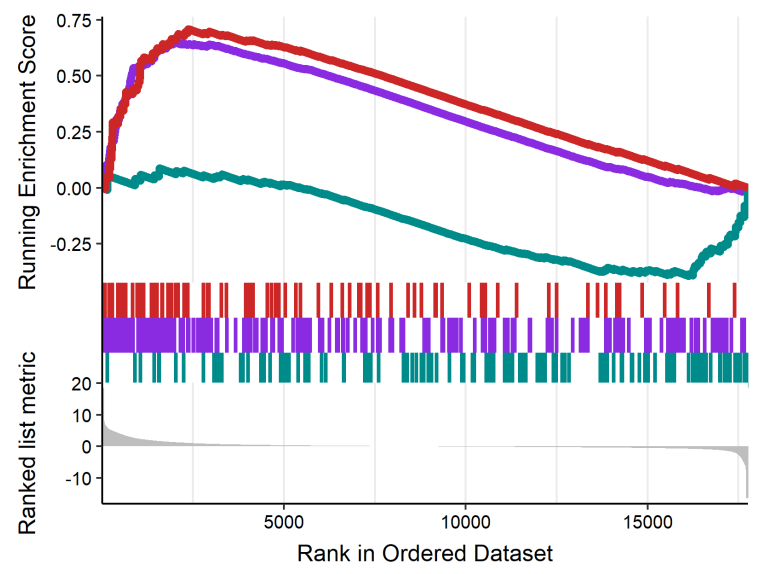

HFD+THCA vs Control

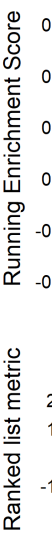
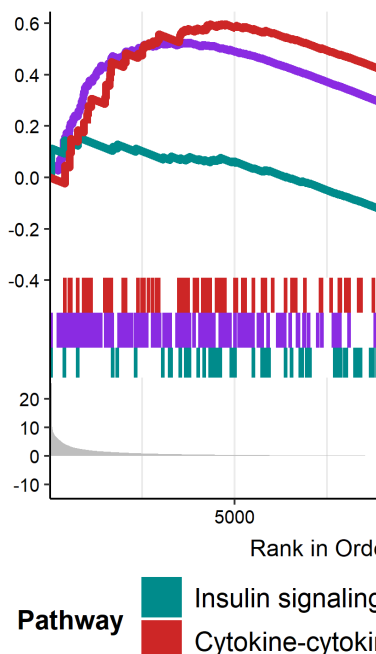


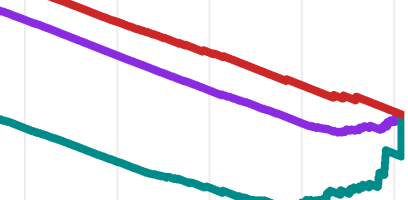

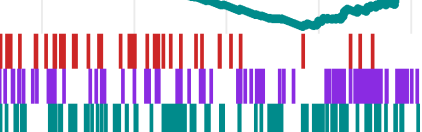

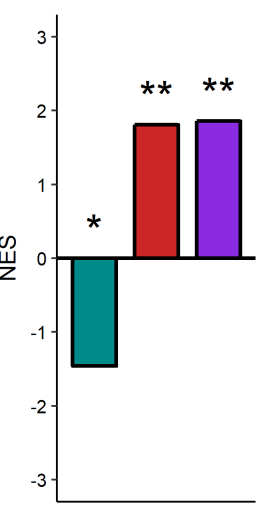

Pathway
HFD+THCA vs Control

525

23

D

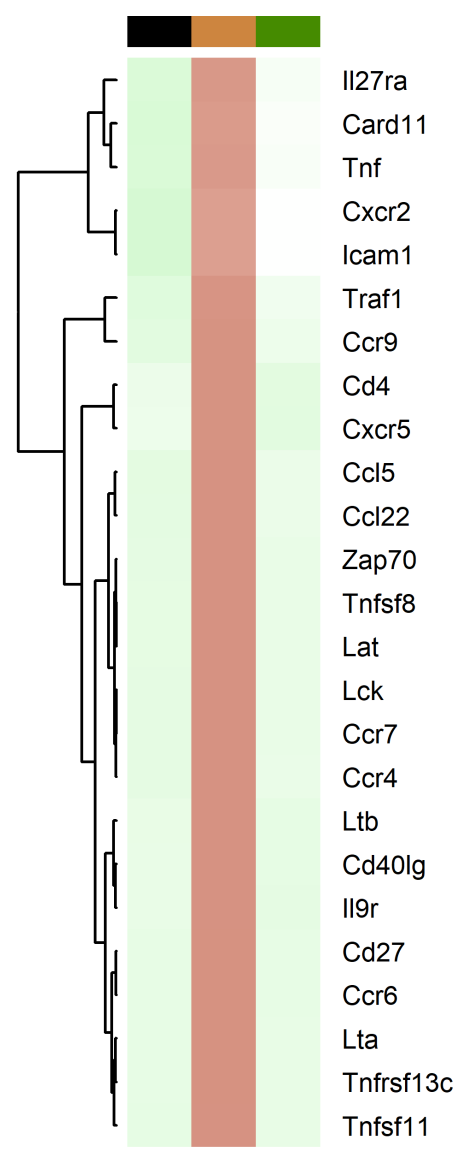


A

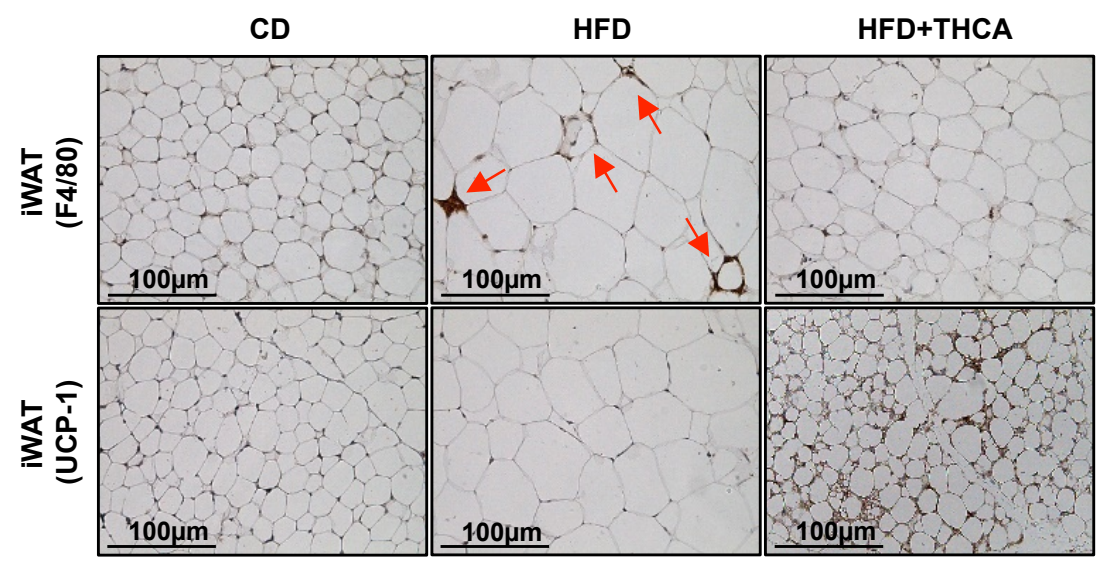

B

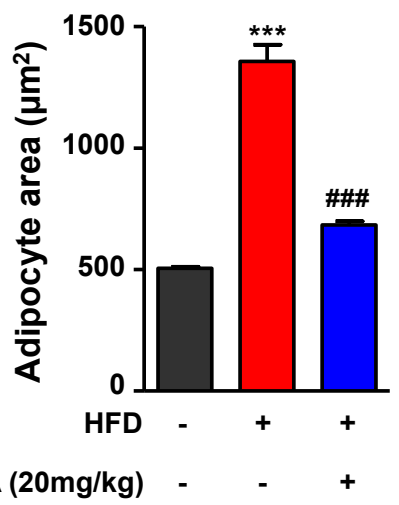

C

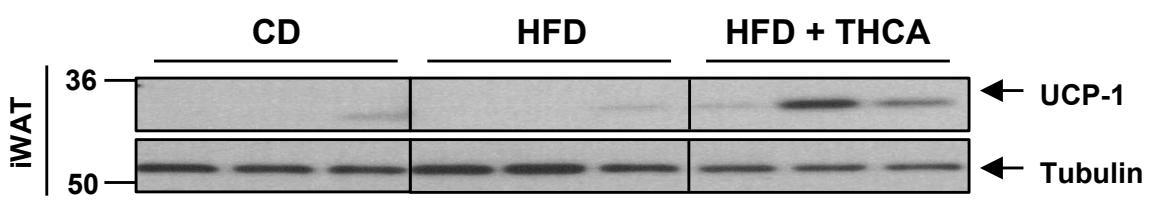

D

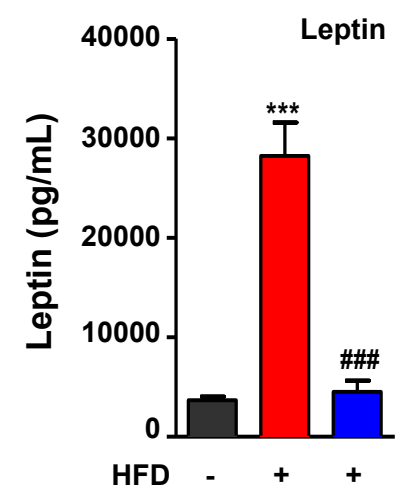

THCA (20mg/kg) - - +

$\mathbf{F}$

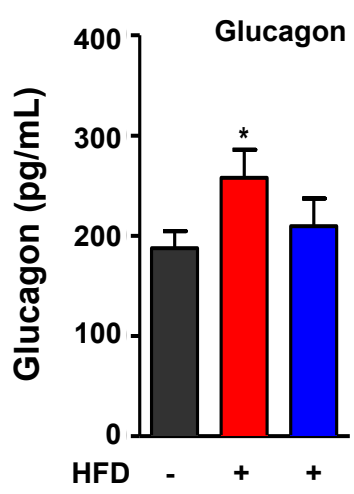

$\mathbf{E}$

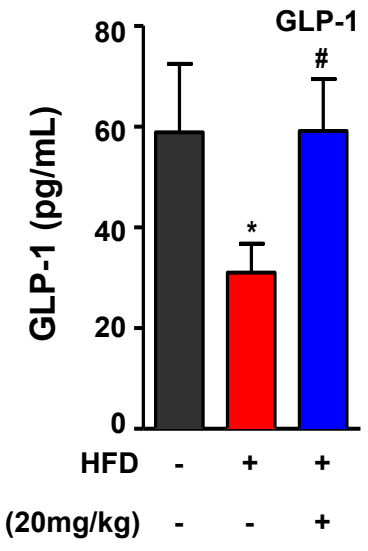

G

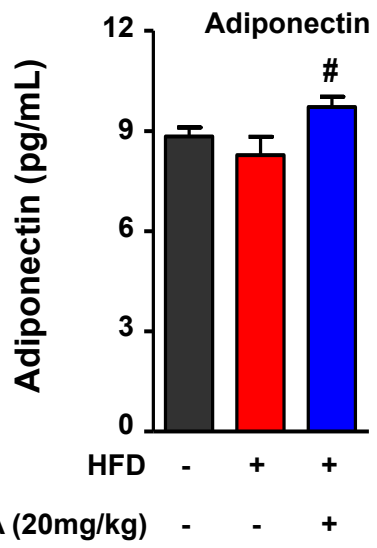

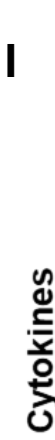

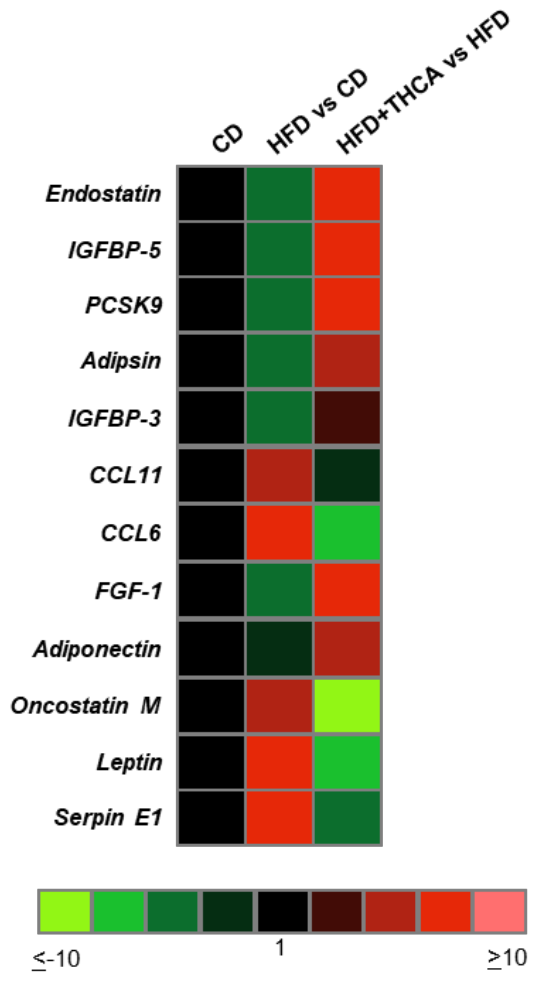

